\title{
Soft and Joint Source-Channel Decoding of Quasi-Arithmetic Codes
}

\author{
Thomas Guionnet \\ Projet TEMICS, IRISA-INRIA, Campus Universitaire de Beaulieu, 35042 Rennes Cedex, France \\ Email: thomas.guionnet@irisa.fr \\ Christine Guillemot \\ Projet TEMICS, IRISA-INRIA, Campus Universitaire de Beaulieu, 35042 Rennes Cedex, France \\ Email: christine.guillemot@irisa.fr \\ Received 20 November 2002; Revised 7 August 2003; Recommended for Publication by Antonio Ortega
}

\begin{abstract}
The issue of robust and joint source-channel decoding of quasi-arithmetic codes is addressed. Quasi-arithmetic coding is a reduced precision and complexity implementation of arithmetic coding. This amounts to approximating the distribution of the source. The approximation of the source distribution leads to the introduction of redundancy that can be exploited for robust decoding in presence of transmission errors. Hence, this approximation controls both the trade-off between compression efficiency and complexity and at the same time the redundancy (excess rate) introduced by this suboptimality. This paper provides first a state model of a quasi-arithmetic coder and decoder for binary and $M$-ary sources. The design of an error-resilient soft decoding algorithm follows quite naturally. The compression efficiency of quasi-arithmetic codes allows to add extra redundancy in the form of markers designed specifically to prevent desynchronization. The algorithm is directly amenable for iterative source-channel decoding in the spirit of serial turbo codes. The coding and decoding algorithms have been tested for a wide range of channel signal-tonoise ratios (SNRs). Experimental results reveal improved symbol error rate (SER) and SNR performances against Huffman and optimal arithmetic codes.
\end{abstract}

Keywords and phrases: robust arithmetic and quasi-arithmetic coding, joint source-channel coding, soft decoding, estimation, MAP.

\section{INTRODUCTION}

Entropy coding, producing variable length codewords (VLCs), is a core component of any data compression scheme. However, VLCs are very sensitive to channel noise: when some bits are altered by the channel, synchronization losses can occur at the receiver, the position of symbol boundaries are not properly estimated, leading to dramatic symbol error rates (SERs). This phenomenon has given momentum to extensive work on the design of procedures for soft decoding and joint source-channel decoding of VLCs. Soft VLC decoding ideas, exploiting residual source redundancy (the so-called excess rate), have also been shown to reduce the "desynchronization" effect as well as the residual bit error rate and SER [1, 2,3]. Models incorporating both VLC-encoded sources and channel codes (CCs) have also been considered $[4,5,6,7]$.

The research effort has first focused on Huffman codes $[3,4,5,8]$ and on reversible VLCs $[6,9,10]$. However, arithmetic codes have gained increased popularity in practi- cal systems, including JPEG2000, H.264, and MPEG-4 standards. Arithmetic coding allows to decouple the coding process from the source model, hence can be used in conjunction with any probabilistic model. A good statistical model of the source is a key element to obtain maximum compression performance. However, the counterpart to the high compression efficiency is an increased sensitivity to noise: a single bit error causes the internal decoder state to be in error. Methods considered to fight against noise sensitivity consist usually in reaugmenting the redundancy of the bit stream either by introducing an error-correcting code or by inserting dedicated patterns in the chain. Along those lines, the author in [11] reintroduces redundancy in the form of parity check bits embedded into the arithmetic coding procedure. A probability interval not assigned to a symbol of the source alphabet or markers inserted at known positions in the sequence of symbols to be encoded are exploited for error detection in $[12,13,14]$. This capability can then be coupled with an automatic request for retransmission (ARQ) procedure $[13,15]$ or used jointly with an error-correcting 
code [16]. Sequential decoding of arithmetic codes is investigated in [17] for supporting error correction capabilities. The complexity of this approach is reduced in [18] by using trellis-coded modulation combined with a list Viterbi decoding algorithm. A soft decoding procedure is described in [19]. However, one difficulty comes from the fact that the code tree, hence the state-space dimension, or the number of states of the model grows exponentially with the number of symbols being encoded. A pruning technique is then used to limit the complexity within a tractable and realistic range. However, it brings inherent limitations when using the decoder in an iterative source-channel decoding structure. The pruning of the tree to limit the complexity is such that, in this particular case, the iterations do not bring a significant gain.

A fast arithmetic coding procedure called quasi-arithmetic coding has been introduced in [20]. It operates on an integer interval $[0, T[$ and on integer subdivisions of this interval. This amounts to approximate the source distribution. This controlled approximation allows to reduce the number of possible coder states without significantly degrading the compression performance. The trade-off between the statespace dimension and the source distribution approximation is controlled by the parameter $T$. If $T$ is sufficiently small, all state transitions and outputs can then be precomputed and table lookups be in turn substituted for arithmetic operations. A quasi-arithmetic coder can be regarded as an arithmetic coder governed by an approximation of the real source distribution.

In this paper, we first revisit finite-state automaton modeling of quasi-arithmetic coding and decoding processes for $M$-ary sources. Notice that the $M$-ary source could be coded directly with a quasi-arithmetic coder. An accurate approximation of the $M$-ary source distribution would however require to set the parameter $T$ to a high value, resulting in a high state-space dimension, hence in high decoding complexity. To maintain the complexity within a tractable range, one would have to rely on a very coarse source distribution approximation. One can instead first map the $M$-ary source model into a binary model by means of a fixed-length binary code represented as a binary tree with symbols at the leaves. These trees are connected up to a depth function of the source model (e.g., for an order-1 Markov source, the depth is one). Leaves of the tree represent terminated symbols and are identified with the root of the next tree. The resulting finite binary tree can be regarded as a stochastic automaton that models the source symbol distribution. Once the $M$-ary source has been converted into a binary source, the latter can be encoded by a quasi-arithmetic coder. The transitions on the binary model govern the quasi-arithmetic coder. The design of an efficient estimation procedure based on the BCJR algorithm [21] follows quite naturally. The decoding complexity remains within a realistic range without the need for applying any pruning of the estimation trellis. The estimation algorithm has been validated under various channel conditions and for different levels of source correlation. Experimental results have shown very high error resilience while at the same time preserving a very good com- pression efficiency. For a comparable overall rate, in comparison with Huffman codes, better compression efficiency of quasi-arithmetic codes allows to dedicate extra redundancy (short "soft" synchronization patterns) specifically to decoder resynchronization, resulting in significantly higher error resilience. The usage of CCs is also considered in order to reduce the bit error rate seen by the source estimation algorithm. The latter can then be placed in an iterative decoding structure in the spirit of serially concatenated turbo codes, provided that the channel decoder and the quasi-arithmetic decoder are separated by an interleaver. Since, in contrast with optimal arithmetic coding, the estimation can be performed without pruning the trellis, the potential of the iterative decoding structure can be fully exploited, resulting in a very low SER (significantly lower than what can be obtained with Huffman codes). Overall, the great flexibility that quasiarithmetic codes offer for adjusting compression efficiency, error resilience, and complexity allows an optimal adaptation to various transmission conditions and terminal capability requirements.

The rest of the paper is organized as follows. Section 2 describes the notations we use and states the problem addressed. Sections 3 and 4 review the principles of arithmetic and quasi-arithmetic coding. Sections 5 and 6 address modeling issues of, respectively, coding/decoding processes and of the source. This material is exploited in the sequel (Sections 7 and 8) for explaining the estimation algorithm and the soft synchronization procedure. Section 9 outlines the construction of the iterative joint source-channel decoding procedure based on quasi-arithmetic codes. Finally, experimental results are described in Section 10. We first compare the performance of the algorithm in terms of SER and signal-to-noise ratio (SNR) with respect to soft Huffman and arithmetic decoding with theoretical Gauss-Markov sources. Simulations results of the joint source-channel turbo decoding algorithm in comparison with soft decoding of quasi-arithmetic codes are also provided.

\section{NOTATIONS AND PROBLEM STATEMENT}

Let $A=A_{1} \cdots A_{L}$ be a sequence of quantized source symbols taking their values in a finite alphabet $\mathscr{A}$ composed of $M=2^{q}$ symbols, $\mathscr{A}=\left\{a_{1}, a_{2}, \ldots, a_{i}, \ldots, a_{M}\right\}$. The sequence $A=A_{1} \cdots A_{L}$ is assumed to form an order-1 Markov chain. This sequence of $M$-ary symbols is converted into a sequence of binary symbols $S=S_{1} \cdots S_{K}$, where $K=q \times L$. This binary source is in turn coded into a sequence of information bits $U=U_{1} \cdots U_{N}$ by means of a quasi-arithmetic coder as depicted in Figure 1. The length $N$ of the information bit stream is a random-variable function of $S$, hence of $A$. The bit stream $U$ is sent over a memoryless channel and received as measurements $Y$; so the problem we address consists in estimating $A$, given the observed values $y$. Notice that we reserve capital letters to random variables and small letters to values of these variables. For handling ranges of variables, we use the notation $X_{u}^{v}=\left\{X_{u}, X_{u+1}, \ldots, X_{v}\right\}$ or $\bar{X}_{I}$, where $I$ is the index set $\{u, u+1, \ldots, v\}$. 


\begin{tabular}{|c|c|c|c|c|}
\hline & \multirow{2}{*}{$\begin{array}{c}M \text {-ary to } \\
\text { binary source } \\
\text { conversion }\end{array}$} & $e$ & \multirow{2}{*}{$\begin{array}{l}\text { Quasi- } \\
\text { arithmetic } \\
\text { coding }\end{array}$} & \\
\hline & & & & $U=U_{1}$ \\
\hline
\end{tabular}

FIGURE 1: Conversions taking place in the coding chain.

\section{ARITHMETIC CODING PRINCIPLES}

We first review the principle of arithmetic coding on a simple example of a source taking values in the alphabet $\left\{a_{1}, a_{2}, a_{3}, a_{4}\right\}$ with the stationary distribution $\mathbb{P}_{a}=$ $[0.6,0.2,0.1,0.1]$. The interval $[0,1[$ is partitioned into four cells representing the four symbols of the alphabet. The size of each cell is the stationary probability of the corresponding symbol. The partition (hence the bounds of the different segments) of the unit interval is given by the cumulative stationary probability of the alphabet symbols. The interval corresponding to the first symbol to be encoded is chosen. It becomes the current interval and is again partitioned into different segments. The bounds of the resulting segments are driven by the model of the source. Considering an order-1 Markov chain, these bounds will be governed by $\mathbb{P}\left(A_{l+1} \mid A_{l}\right)$, hence, in this particular case, will be function of both the probability of the previous symbol and of the cumulative probability of the alphabet symbols. Therefore, the arithmetic coder adapts in this case to the entropy rate $H\left(A_{l+1} \mid A_{l}\right)$ of process $A$, that is, it compresses the innovation of the Markov chain $A$.

In the example above, when the sequence $a 1, a 4, a 2, a 1$ has been encoded, the current interval is [0.576, 0.5832[. Any number in this interval can be used to identify the sequence. We consider 0.576 . The decoding of the sequence is performed by reproducing the coder behavior. First, the interval $[0,1$ [ is partitioned according to the cumulative probability of the source. Since the value 0.576 belongs to the interval $[0,0.6[$, it is clear that the first symbol encoded has been $a 1$. Therefore, the first symbol is decoded and the interval [0, 0.6[ is partitioned according to the cumulative probability of the source. The process is repeated until full decoding of the sequence. Practical implementations of arithmetic coding have been first introduced in $[22,23]$ and developed further in [24]. One problem that may arise when implementing arithmetic coding is the high precision needed to represent very small real numbers. In order to overcome this difficulty, one can base the algorithm on the binary representation of real numbers in the interval $[0,1[$ (see [25]). Any number in the interval [0,0.5 [ will have its first bit equal to 0 , while any number in the interval $[0.5,1$ [ will have its first bit equal to 1 . Therefore, during the encoding process, as soon as the current interval is entirely under or over $1 / 2$, the corresponding bit is emitted and the interval length is doubled. There is a specific treatment for the intervals straddling $1 / 2$. When the current interval straddles $1 / 2$ and is in $[0.25,0.75$ [, it cannot be identified by a unique bit. Its size is therefore doubled without emitting any bit, and the number of rescaling operations taking place before emitting any bit is memorized. When reaching an interval for which one bit $U_{i}=u_{i}$ can be emitted, then this bit will be followed by a number of bits $U_{i+1}=u_{i+1} \cdots U_{i+n}=u_{i+1}$, where $n$ is the number of scaling operations that have been performed before the emission of $U_{i}$, and where $u_{i+1}=u_{i}+1 \bmod 2$. The use of this technique guarantees that the current interval always satisfies low $<0.25<0.5 \leq$ high or low $<0.5<0.75 \leq$ high, where low and high are, respectively, the lower and upper bounds of the current interval. This avoids the problems of precision which may otherwise occur in the case of small intervals straddling the middle of the segment $[0,1[$.

\section{FAST-REDUCED PRECISION IMPLEMENTATION}

Arithmetic coding is near optimality in terms of compression. Error-resilient decoding solutions can be designed [19], however, their complexity can be an issue in some contexts. The coding process can indeed be modeled under the form of a stochastic automaton, where the states are defined by three variables: low, up (denoting the bounds of the subinterval resulting from successive subdivisions of the interval $[0,1[)$, and $n \mathrm{scl}$ denoting the number of scalings performed since the last emitted bit, as explained in Section 3. Subdivisions of the interval [low, up[, hence next states, are functions of the cumulative probability distribution of the source. Without the source model, the possible number of subdivisions, hence of states, may be infinite. If the source distribution is known, the number of states still grows exponentially with the number of symbols being encoded.

\subsection{Quasi-arithmetic coding}

It is observed in [20] that controlled approximations can reduce the number of possible states without significantly degrading compression performance. All state transitions and outputs can then be precomputed and table lookups can be used instead of arithmetic operations. This fast, but reduced precision, implementation of arithmetic coding is called quasi-arithmetic coding [20]. Instead of using the real interval $[0,1[$, quasi-arithmetic coding is performed on an integer interval $[0, T[$. The value of $T$ controls the trade-off between complexity and compression efficiency: if $T$ is sufficiently large, then the interval subdivisions will follow closely the distribution of the source. In contrast, if $T$ is small, all the interval subdivisions can be precomputed.

Given the $M$-symbol alphabet $\mathscr{A}$, the sequence of symbols $A_{1}^{L}$ is translated into a sequence of bits $U_{1}^{N}$ by an $M$-ary decision tree. This tree can be regarded as an automaton that models the bit stream distribution. The encoding of a symbol determines the choice of a vertex or branch in the tree. Each node of the tree identifies a state $X$ of the arithmetic coder and to each transition can be associated the emission of a sequence of bits of variable lengths. Successive branching 
TABLE 1: Quasi-arithmetic coder states, transitions, and outputs for $T=4$.

\begin{tabular}{|c|c|c|c|c|c|c|c|c|c|c|c|}
\hline \multirow{3}{*}{ State $X_{l}$} & \multirow{3}{*}{$\operatorname{low} A_{l}$, up $A_{l}[$} & \multirow{3}{*}{\multicolumn{2}{|c|}{$\mathbb{P}(0)$ (corresponding interval subdivision) }} & \multicolumn{4}{|c|}{ Normal state model } & \multicolumn{4}{|c|}{ Simplified state model } \\
\hline & & & & \multicolumn{2}{|c|}{0} & \multicolumn{2}{|c|}{1} & \multicolumn{2}{|c|}{ MPS } & \multicolumn{2}{|c|}{ LPS } \\
\hline & & & & Out & Next & Out & Next & Out & Next & Out & Next \\
\hline \multirow{3}{*}{0 (start) } & \multirow{3}{*}{$0,4[$} & $0.63 \leq \mathbb{P}(0)$ & $([0,3[)$ & - & 1 & 11 & 0 & - & 1 & 11 & 0 \\
\hline & & $0.37 \leq \mathbb{P}(0)<0.63$ & $([0,2[)$ & 0 & 0 & 1 & 0 & 0 & 0 & 1 & 0 \\
\hline & & $\mathbb{P}(0)<0.37$ & $([0,1[)$ & 00 & 0 & - & 2 & - & - & - & - \\
\hline \multirow{2}{*}{1} & \multirow{2}{*}{$0,3[$} & $0.5 \leq \mathbb{P}(0)$ & $([0,2[)$ & 0 & 0 & 10 & 0 & 0 & 0 & 10 & 0 \\
\hline & & $\mathbb{P}(0)<0.5$ & $([0,1[)$ & 00 & 0 & $f$ & 0 & - & - & - & - \\
\hline \multirow{2}{*}{2} & \multirow{2}{*}{$1,4[$} & $0.5 \leq \mathbb{P}(0)$ & $([1,3[)$ & $f$ & 0 & 11 & 0 & - & - & - & - \\
\hline & & $\mathbb{P}(0)<0.5$ & $([1,2[)$ & 01 & 0 & 1 & 0 & - & - & - & - \\
\hline
\end{tabular}

on the tree (or transitions between states) follow the distribution of the source $\left(\mathbb{P}\left(A_{l} \mid A_{l-1}\right)\right.$ for an order-1 Markov source or $\mathbb{P}\left(A_{l}\right)$ in the zeroth-order case). Let $X_{l}$ denote the state of the automaton at each symbol instant $l$. As in the case of optimal arithmetic coding, the state $X_{l}$ of the quasiarithmetic coder is defined by three variables: low $A_{l}$, up $A_{l}$, and $n \mathrm{scl}_{l}$. The terms low $A_{l}$ and up $A_{l}$ denote the bounds of the subinterval resulting from successive subdivisions of the interval $\left[0, T\left[\right.\right.$ triggered by the encoding of the sequence $A_{1}^{l}$. The quantity $n \mathrm{scl}_{l}$ is (re)set to zero when a bit is emitted and incremented each time a rescaling takes place. Hence, this quantity denotes the number of scalings performed since the last emitted bit. When a bit is emitted, it is followed by $n \mathrm{scl}_{l}$ bits of opposite value (see Section 3).

Since there is a finite number of possible integer subdivisions of the interval $[0, T[$, all the possible states of the quasi-arithmetic coder can be precomputed without knowledge of the source. This is however without accounting for the variable $n \mathrm{scl}_{l}$. Indeed, the variable $n \mathrm{scl}_{l}$ is not bounded. The solution is then to consider $n \mathrm{scl}_{l}$ as a variable resulting from state transitions (output variable) and not to consider this variable in the precomputation of the coder states. Table 1 gives the states, outputs, and all possible transitions of a quasi-arithmetic coder precomputed for a binary source with $T=4$. The value of the variable $n \mathrm{scl}_{l}$ is not considered in this state model. Only the action of incrementing this variable when a rescaling is taking place is signalled by the letter $f$ in the table, also referred to as follow up in [26]. The coder has three states corresponding to integer subdivisions of the interval $[0,4[$. The subdivisions that can possibly take place next are functions of the source probability distribution. They are chosen in such a way that the corresponding distribution approximation will minimize the excess rate [26]. For example, we assume that the automaton is in state $X_{l}=1$ (defined by the interval [low $A_{l}$, up $A_{l}[=[0,3[$ ). Depending on the probability of the input binary symbol 0 , the interval $[0,3[$ will be further subdivided into $[0,2[$ corresponding to an approximated probability of $2 / 3$ if its probability is higher than $1 / 2$, or into $[0,1$ [ corresponding to an approximated probability of $1 / 3$ if its probability is lower than $1 / 2$. Both subdivisions result, after appropriate bit emission and scaling, into the state 0 . The number of possi- ble states $X_{l}$ is $3 T^{2} / 16$. If we take into account the different source distributions, the number of possible transitions from all the states $X_{l}$ is $9 T^{3} / 64-6 T^{2} / 32+T / 4$.

The number of states can be further reduced by identifying the symbols as more probable (MPS) and less probable (LPS) rather than as 1 and 0 . This amounts to reducing the number of possible combinations of the binary source probabilities, the MPS being either the symbol 0 or the symbol 1 . This allows to combine transitions and eliminate states as shown in Table 1 . The sequence $X_{0} \cdots X_{L}$ is a Markov chain and the output of the coder is the function of transitions of this chain. This state representation can help designing a robust maximum a posteriori (MAP) decoder. For a deeper understanding of quasi-arithmetic coding, the reader may refer to $[20]$.

\subsection{Quasi-arithmetic decoding}

We first consider the operation of an optimum arithmetic decoder. A sequence of arithmetically coded bits $U_{1}^{N}$ is translated into a sequence of symbols $A_{1}^{L}$ by a binary decision tree. Each bit determines the choice of a vertex in the tree. Each node $v$ of the tree identifies a state of the arithmetic decoder and corresponds to a tuple $U_{1}^{n-1}$ from which two transitions are possible: $U_{n}=0$ or $U_{n}=1$. The state of the decoder is specified by two intervals: [low $U_{n}$, up $U_{n}$ [ and [low $A_{L_{n}}$, up $A_{L_{n}}$ [. The interval [low $U_{n}$, up $U_{n}$ [ defines the segment of the interval $[0,1$ [ selected by a given input bit sequence $U_{1}^{n}$. The interval [low $A_{L_{n}}$, up $A_{L_{n}}$ [ relates to the subdivision obtained when the symbol $A_{L_{n}}$ can be decoded without ambiguity. Both intervals must be scaled appropriately in order to avoid numerical precision problems. However, in contrast to the coding process, there is no need to keep track of the scalings that have been performed.

We now consider the interval $[0, T[$ and finite interval subdivisions. The quasi-arithmetic decoder can also be expressed in the form of an automaton. Let $X_{n}$ be its state at bit instant $n ; X_{n}$ stores the four variables [low $U_{n}$, up $U_{n}$ [ and [low $A_{L_{n}}$, up $A_{L_{n}}$ [. Since there is a finite number of possible subdivisions of the interval $[0, T[$, there is a finite number of states for the quasi-arithmetic decoder which can be pre-computed. Table 2 gives the states, transitions, and outputs of the quasi-arithmetic decoder for a binary source 
TABle 2: Quasi-arithmetic decoder states, transitions, and outputs for $T=4$.

\begin{tabular}{c|c|cc|cccc}
\hline \multirow{2}{*}{ State $X_{n}$} & State variables & \multicolumn{2}{|c|}{$\mathbb{P}\left(\right.$ MPS) (corresponding subdivision of $\left[\right.$ low $A_{L_{n}}$, up $A_{L_{n}}[$ ) } & \multicolumn{2}{c|}{$U_{n}=0$} & \multicolumn{2}{c}{$U_{n}=1$} \\
Out & Next & Out & Next \\
\hline \multirow{2}{*}{0 (start) } & {$\left[\right.$ low $U_{n}$, up $U_{n}[=[0,4[$} & $0.63 \leq \mathbb{P}(\mathrm{MPS})$ & $([0,3[)$ & MPS, MPS & 0 & - & 1 \\
& {$\left[\right.$ low $A_{L_{n}}$, up $A_{L_{n}}[=[0,4[$} & $0.37 \leq \mathbb{P}(\mathrm{MPS})<0.63$ & $([0,2[)$ & MPS & 0 & LPS & 0 \\
\hline \multirow{2}{*}{1} & $\begin{array}{l}\text { low } U_{n}, \text { up } U_{n}[=[2,4[ \\
{\left[\text { low } A_{L_{n}}, \text { up } A_{L_{n}}[=[0,4[\right.}\end{array}$ & $0.63 \leq \mathbb{P}(\mathrm{MPS})$ & $([0,3[)$ & MPS, LPS & 0 & LPS & 0 \\
\hline
\end{tabular}

and $T=4$, with the MPS/LPS simplification. The decoder in this particular example has two states. Further subdivisions that will lead to transitions to next states are functions of the source probability distribution (e.g., $\mathbb{P}(\mathrm{MPS})$ in Table 2). We assume, for example, that the automaton is in state $X_{n}=0$ (defined by the two intervals [low $U_{n}$, up $U_{n}$ [ = $\left[0,4\left[\right.\right.$ and [low $A_{L_{n}}$, up $A_{L_{n}}[=[0,4[)$, and that the input is $U_{n}=0$. Depending on the probability of the source to be coded (hence here of the MPS and LPS symbols), the interval $[0,4$ [ will be further subdivided into [0,3[ (if MPS probability is higher than 0.63 ) or into [0,2[ (if MPS probability is lower than 0.63 ), both subdivisions resulting into the state 0 .

\subsection{Source distribution approximation}

Arithmetic coding realizes a conversion of source distributions: in the general case, it realizes a conversion of a sequence of symbols of an $M$-ary source of a given distribution into a sequence of symbols of a binary source with an independent and uniform distribution. A quasi-arithmetic coder does not produce a sequence of independently and uniformly distributed bits due to the approximation of the source distribution that it realizes. It has been shown in [26] that this approximation does not induce a significant increase in code length. This however depends on the source statistics. This statement is true if the symbol probabilities are comprised between $1 / t$ and $(t-1) / t$, where $t$ is the width of the current interval to be subdivided. Hence, the choice of the value of $T$ may depend on the source distribution. The excess rate resulting from the approximation can be computed as follows. Let $a$ be a symbol taking its value in $\mathscr{A}, \mathbb{P}(a)$ the probability of the event $a$, and $\mathbb{Q}(a)$ the approximation of $\mathbb{P}(a)$ made by the quasi-arithmetic coder. The entropy of the source is given by

$$
H=-\sum_{a=a_{1}}^{a_{M}} \mathbb{P}(a) \log _{2} \mathbb{P}(a)
$$

The performance of the quasi-arithmetic coder can then be measured by

$$
R=-\sum_{a=a_{1}}^{a_{M}} \mathbb{P}(a) \log _{2} \mathbb{Q}(a) .
$$

The excess rate induced by the quasi-arithmetic coder can hence be expressed as

$$
\begin{aligned}
E & =R-H \\
& =\sum_{a=a_{1}}^{a_{M}} \mathbb{P}(a) \log _{2} \frac{\mathbb{P}(a)}{\mathbb{Q}(a)} \\
& =D(\mathbb{P} \| \mathbb{Q}),
\end{aligned}
$$

where $D(\mathbb{P} \| \mathbb{Q})$ is the Kullback-Leibler distance or relative entropy between the approximate distribution $\mathbb{Q}$ and the true distribution $\mathbb{P}$.

\section{SOURCE MODEL}

For a binary source, the variable $T$ can be limited to a small value (down to 4) at a small cost in terms of compression [26]. This motivates a conversion of the $M$-ary source into a binary source to be then encoded by the quasi-arithmetic coder. This conversion amounts to a fixed-length binary coding of the source.

We first assume that the source quantized on $M=2^{q}$ symbols is an order-0 Markov source. It can then be represented by a binary tree of depth $q$, as shown in Figure 2a for $M=4$. The transition probabilities on the binary tree can be computed easily from the distribution of the source. The resulting binary tree can be seen as an automaton that models the $M$-ary source stationary distribution. A complete model of the source can be obtained by connecting the successive local models. One possible solution consists in identifying the leafnodes of the binary tree with the rootnode of the next tree. This leads to the three states automaton of Figure $2 \mathrm{~b}$ in the particular case of an order-0 Markov source with $M=4$. In Figure 2c, the same automaton is shown in the form of a trellis, with the probability of each bit indicated on the corresponding transition. Relying on this stochastic automaton model, the sequence of the resulting binary symbols can be modeled as a function of a hidden Markov model. Let $C_{k}$ denote the state $v$ of the automaton after $k$ binary symbols have been produced. The sequence $C_{0}, \ldots, C_{K}$ is a Markov chain, and the resulting sequence of binary symbols is a function of transitions of this chain, that is, $S_{k}=\phi\left(C_{k-1}, C_{k}\right)$.

We now assume that the source is an order-1 Markov source. To take into account the correlation present in the $M$-ary source, in the model construction, one must in addition keep track of the last $M$-ary symbol coded. In order to do so, one could define the state variable $C_{k}$ as a pair $(\nu, \sigma)$, where $\sigma$ is the value of the last completed symbol $A_{l}$ and $v$ is the current state of the stochastic automaton describing the construction of the next $M$-ary symbol, following 


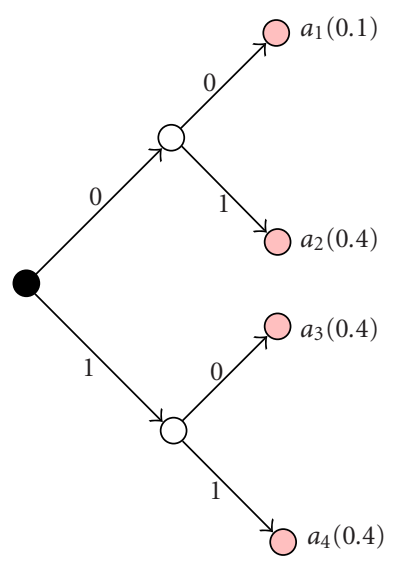

(a)

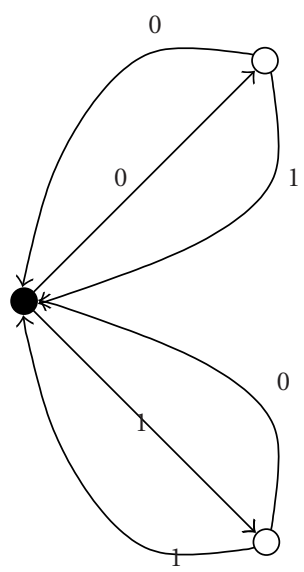

(b)

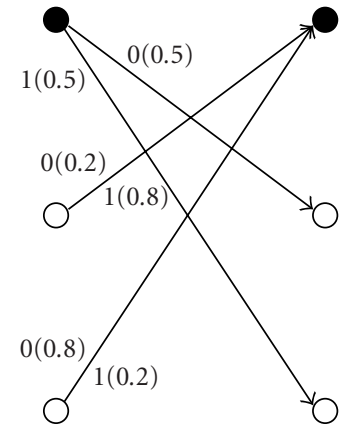

(c)

FIGURE 2: Graphical representation under the form of (a) a tree, (b) a stochastic automaton, and (c) a trellis of the binary model of the order-0 Markov source. Black dots correspond to leafnodes identified to rootnodes of next trees. White dots correspond to intermediate nodes of the binary representation of the $M$-ary symbols $(M=4)$.

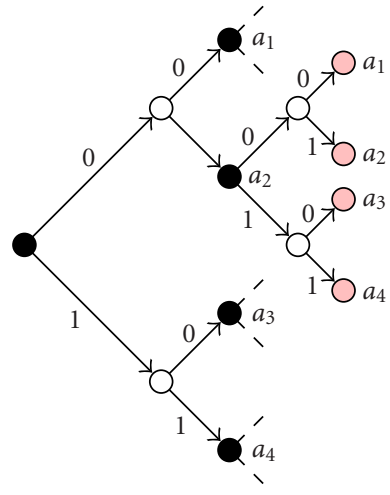

(a)

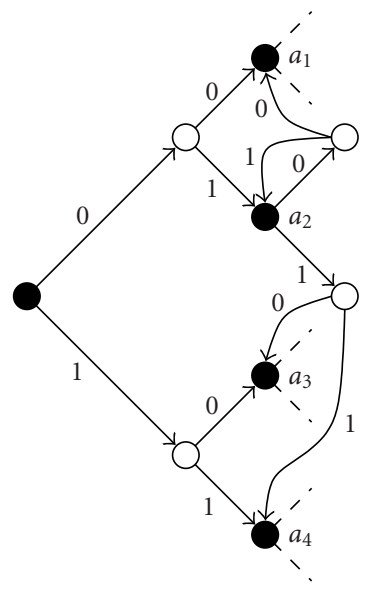

(b)

FIGURE 3: Graphical representation under the form of (a) a tree and (b) a stochastic automaton of the binary model of the order-1 Markov source $(M=4)$.

$\mathbb{P}\left(A_{l+1} \mid A_{l}\right)$. Alternately, one can take into account the conditional distribution $\mathbb{P}\left(A_{l+1} \mid A_{l}\right)$, by connecting $M+1$ trees of depth $q$ as shown in Figure 3a, and define the state variable $C_{k}$ as a node $v$ in the resulting tree. The complete model of the source is then obtained by additional transitions from leaves to intermediate nodes as shown in Figure 3b. In this particular case, the model leads to a state space of dimension 15 . The state-space dimension for an order- $n$ Markov source quantized on $M$ symbols is given by $M^{n+1}-1$. For both state models $\left(C_{k}=(\nu, \sigma)\right.$ and $\left.C_{k}=(\nu)\right)$, the sequence $C_{1} \cdots C_{K}$ is a Markov process, the transitions of which produce the sequence $S$ of binary symbols.

Remark 1 . The construction of the binary model, that is, the assignment of the binary codewords to the different sym- bols, has a strong impact on the reliability of the estimation. The transition probabilities on the binary tree are indeed defined by this symbol-to-leave assignment. Increased estimation reliability can be obtained when the different paths (or branches) on the model have a highly nonuniform likelihood, that is, when the uncertainty of some branches is much lower than for others. The assignment of binary codewords to symbols must hence be such that the expression $\sum_{X} \mathbb{P}(X) H(X)$ is minimized, where $\mathbb{P}(X)$ is the probability of the state $X$ of the model and where $H(X)$ is the entropy of the transitions leaving $X$. For small source alphabets, the minimization can be achieved by a simple exhaustive search approach. However, for larger size alphabets, the exhaustive search among all possible index assignments rapidly becomes untractable. This complexity can be reduced by limiting the 
search space to a subset of index assignments. In the experiments reported here, this subset has been obtained by a simple circular shift of an initial index assignment. This initialization has an impact on the resulting SER and SNR performances. A lexicographic index assignment to symbols ranked by decreasing probability values turned out to provide good performance.

\section{MODELING BIT STREAM DEPENDENCIES}

In order to design efficient algorithms for estimating the sequence of symbols that has been emitted, one has now to build a model of bit stream dependencies. For this, we consider the product (in the sense of product on automata or of tensor product of stochastic models) of the source and coder or source and decoder models. Estimation algorithms can be defined for both models. However, in Section 7, the estimation is performed only on the product of the source and decoder models, since with the source and coder models, handling the $n \mathrm{scl}_{k}$ variable can increase dramatically the number of states. For the source, in the sequel, we consider the binary source model described in Section 5.

\subsection{Product model: source and coder}

The state of the product system must gather state information of two automata: the automaton modeling the source distribution and the coder model. Hence, the state $X_{k}$ of the product system is defined as $X_{k}=\left(\right.$ low $S_{k}$, up $\left.S_{k}, C_{k}\right)$. One could expect the dimension of the resulting state space to be the product of dimensions of the states spaces of the two models (source and coder). However, simplifications occur. Again, this is better explained with the simple source and coder examples of Table 1 and Figure 2.

The system resulting from the product of the coder of Table 1 and the order-0 Markov source model of Figure 2c is illustrated in Table 3 and Figure 4, respectively. For $T=4$ and using the MPS/LPS simplification, the transitions do not lead to rescalings of the interval [low $S_{k}$, up $S_{k}$ [. For different values of $T$, rescaling of the interval could occur and would then be signaled with the same notation $f$ as in Table 1. Since the coder model has 2 states and the binary source model has 3 states, the dimension of the resulting state space should in principle be 6 . However, it turns out that in general, fewer states are necessary (only 4 states instead of 6 in the simple coder and source examples of Table 1 and Figure 2). This simplification results from the fact that the transitions in the coder model are a function of the source probability distribution. Depending on the stationary probabilities of the input binary source, the general coder model given in Table 1 simplifies as shown in Figure 5. The probabilities of the binary symbols resulting from the conversion of the $M$-ary source depend on the previous state of the source model. Therefore, transitions on the source model will trigger the use of one of the two quasi-arithmetic trellis of Figure 5. In the example of Figure 5b, it can be verified easily that some states will never be reached, hence reducing the state-space dimension from 6 to 4 .
TABle 3: Source-coder product model: states, outputs, and transitions.

\begin{tabular}{c|c|c|c}
\hline State & State Variables & MPS & LPS \\
\hline \multirow{2}{*}{0 (start) } & {$[0,4[$} & output: 0 & output: 1 \\
& $C=0$ & next state: 1 & next state: 2 \\
\hline \multirow{2}{*}{1} & {$[0,4[$} & output: - & output: 11 \\
& $C=1$ & next state: 3 & next state: 0 \\
\hline \multirow{2}{*}{2} & {$[0,4[$} & output: - & output: 11 \\
& $C=2$ & next state: 3 & next state: 0 \\
\hline \multirow{2}{*}{3} & {$[0,3[$} & output: 0 & output: 10 \\
& $C=0$ & next state: 1 & next state: 2 \\
\hline
\end{tabular}

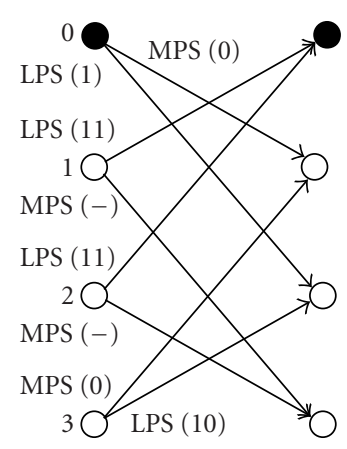

FIGURE 4: Source-coder product model: trellis representation.

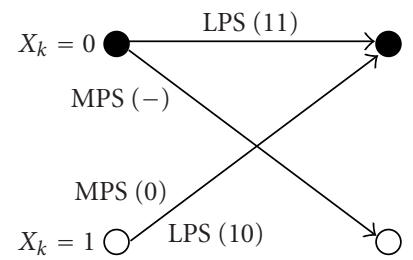

(a)

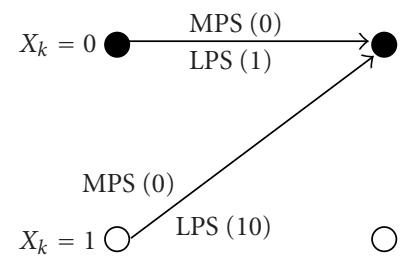

(b)
FIGURE 5: Quasi-arithmetic coder model: (a) $0.63 \leq \mathbb{P}$ (MPS) and (b) $0.5 \leq \mathbb{P}($ MPS $)<0.63$.

In general, the state-space dimension cannot be known a priori; it must be computed, given $T$ and the binary source model. If $N_{C}$ is the number of states of the binary source model, the maximum number of states is $3 T^{2} N_{C} / 16$.

The number of bits produced by each transition on the above model being random, the structure of dependencies between the sequence of measurements and the model states is random. In order to capture this randomness, we actually consider the augmented Markov chain $(X, N)=$ $\left(X_{1}, N_{1}\right) \cdots\left(X_{K}, N_{K}\right)$. This yields the structure of dependencies graphically depicted in Figure 6. Using this model, a sequence of binary symbols $S_{1}^{K}$ is translated into a sequence of bits $U_{1}^{N_{K}}$, where $N_{K}$ is the number of bits emitted when $K$ symbols have been encoded. Given a state $X_{k}$ and an input symbol $S_{k+1}$, the automaton specifies the bits 


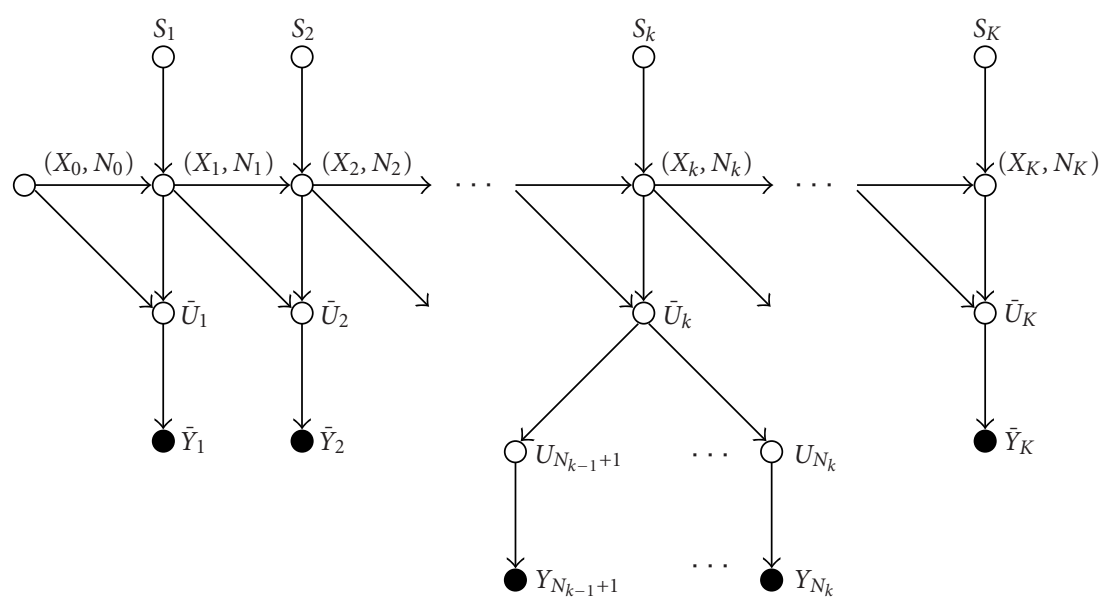

FIGURE 6: Source-coder product model $\left(N_{k} \geq N_{k-1}\right)$. White and black dots represent, respectively, the hidden and observed variables.

$\bar{U}_{k+1}=U_{N_{k}+1}^{N_{k+1}}$ that have to be emitted and the next state $X_{k+1}$. Notice that no bits may be emitted by a transition. The probabilities of successive branchings (e.g., of transitions between $\left(X_{k}, N_{k}\right)=\left(\right.$ low $S_{k}$, up $\left.S_{k}, C_{k}, N_{k}\right)$ and $\left(X_{k+1}, N_{k+1}\right)=$ $\left(\right.$ low $S_{k+1}$, up $\left.S_{k+1}, C_{k+1}, N_{k+1}\right)$ ) in the trellis are given by the binary source model, that is, $\mathbb{P}\left(C_{k+1} \mid C_{k}\right)=\mathbb{P}\left(S_{k+1} \mid C_{k}\right)$. Measurements $\bar{Y}_{k}$ on the bits $\bar{U}_{k}$ are gathered at the output of the transmission channel.

\subsection{Product model: source and decoder}

A product model of source and decoder can be constructed similarly. The state of the product system must gather state information of the source and decoder models. Hence, the state $X_{n}$ of the product system is defined as $X_{n}=\left(\operatorname{low} U_{n}\right.$, up $U_{n}$, low $S_{K_{n}}$, up $\left.S_{K_{n}}, C_{K_{n}}\right)$. The system resulting from the product of the decoder of Table 2 and the simple source model of Figure $2 \mathrm{c}$ is illustrated in Table 4 and Figure 7, respectively. Again, the state space dimension depends on the coder precision parametrized by $T$ and of the source model.

The number of symbols being produced by each transition on the above model is random. Therefore, the structure of dependencies between the sequence of measurements and the sequence of decoded symbols is random. This is handled by considering the augmented Markov chain $(X, K)=\left(X_{1}, K_{1}\right) \cdots\left(X_{N}, K_{N}\right)$ with the structure of dependencies graphically depicted in Figure 8. Using this model, a sequence of bits $U_{1}^{N}$ is translated into a sequence of symbols $S_{1}^{K_{N}}$, where $K_{N}$ is the number of symbols decoded when $N$ bits have been received. Given a state $X_{n}$ and an input bit $U_{n+1}$, the automaton produces the sequence of symbols $\bar{S}_{n+1}=S_{K_{n}+1}^{K_{n+1}}$ and the next state $X_{n+1}$. The probabilities of successive branchings (i.e., of transitions between $\left(X_{n}, K_{n}\right)$ and $\left.\left(X_{n+1}, K_{n+1}\right)\right)$ in the trellis depend on the binary source model and are given by

$$
\prod_{k=K_{n}+1}^{K_{n+1}} \mathbb{P}\left(S_{k} \mid C_{k-1}\right) .
$$

TABle 4: Source-decoder product model: states, outputs, and transitions.

\begin{tabular}{c|c|c|c}
\hline State & State variables & 0 & 1 \\
\hline \multirow{3}{*}{0 (start) } & {$[0,4[$} & output: MPS & output: LPS \\
& {$[0,4[$} & next state: 1 & next state: 2 \\
\hline \multirow{3}{*}{1} & {$[0,4[$} & output: MPS, MPS & output: -0 \\
& {$[0,4[$} & next state: 1 & next state: 3 \\
\hline \multirow{3}{*}{2} & $C=1$ & output: MPS, MPS & output: - \\
& {$[0,4[$} & next state: 1 & next state: 4 \\
\hline \multirow{3}{*}{3} & {$[0,4[$} & output: MPS, LPS & output: LPS \\
& $C=2$ & next state: 2 & next state: 0 \\
\hline \multirow{3}{*}{4} & {$[2,4[$} & output: MPS, LPS & output: LPS \\
& $C=1$ & next state: 2 & next state: 0 \\
\hline
\end{tabular}

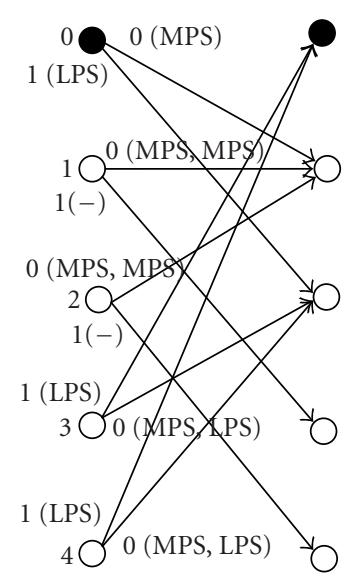

FIGURE 7: Source-decoder product model: trellis representation. 


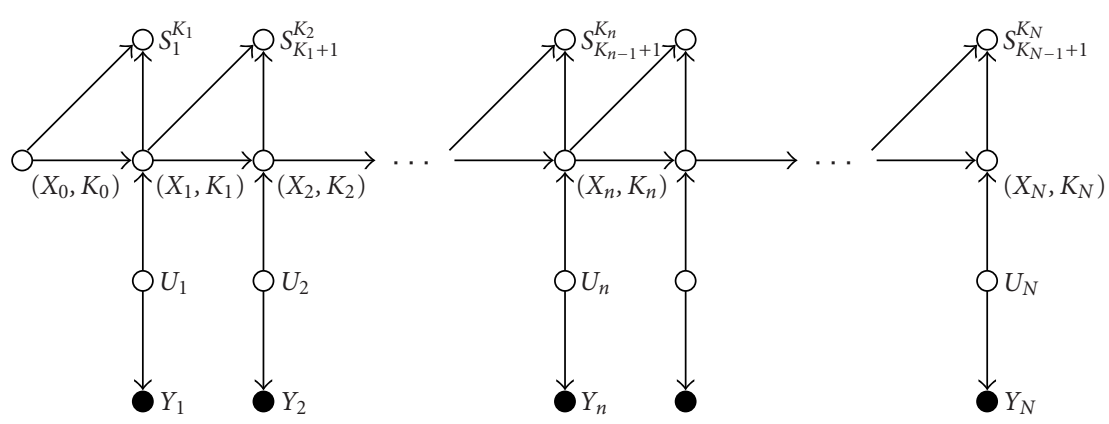

FIGURE 8: Source-decoder product model. White and black dots represent, respectively, the hidden and observed variables.

\subsection{Source distribution approximation}

The entropy of the $M$-ary source computed on the binary model in Section 6 (e.g., Figure 2c) is given by

$$
H(S \mid C)=-\sum_{c=0}^{N_{C}-1} \mathbb{P}(c) \sum_{s=0,1} \mathbb{P}(s \mid c) \log _{2} \mathbb{P}(s \mid c),
$$

where $C$ denotes the state variable of the source model, $c$ the indices of the possible values it can take, and $N_{C}$ the dimension of the state space. The performance of the quasiarithmetic coder when applied on this binary source for a given value of the parameter $T$ can be measured by

$$
R(S \mid X)=-\sum_{x=0}^{N_{X}-1} \mathbb{P}(x) \sum_{s=0,1} \mathbb{P}(s \mid x) \log _{2} \mathbb{Q}(s \mid x)
$$

where $X$ denotes the state variable of the product model (source + coder), $x$ represents the possible indices taken by the variable $X$, and $N_{X}$ is the dimension of the state-space function of $T$. The quasi-arithmetic coder realizes an approximation of the distribution of the binary source, hence of the $M$-ary source. This approximation is, however, for a given value of the parameter $T$, more accurate than when applying the quasi-arithmetic coder directly on the $M$-ary source. Here, the excess rate for a given value of $T$ is given by

$$
\begin{aligned}
E(S \mid X) & =R(S \mid X)-H(S \mid X) \\
& =\sum_{x=0}^{N_{X}-1} \mathbb{P}(x) \sum_{s=0,1} \mathbb{P}(s \mid x) \log _{2} \frac{\mathbb{P}(s \mid x)}{\mathbb{Q}(s \mid x)} \\
& =D(\mathbb{P}(s \mid x) \| \mathbb{Q}(s \mid x)),
\end{aligned}
$$

where $D(\mathbb{P}(s \mid x) \| \mathbb{Q}(s \mid x))$ is the conditional relative entropy between theapproximate conditional distribution $\mathbb{Q}$ and the true conditional distribution $\mathbb{P}$. One may notice that several states of the quasi-arithmetic coder can correspond to a given state $c$ of the binary source model. Hence, several different approximations $\mathbb{Q}(s \mid x)$ of $\mathbb{P}(s \mid c)$ can exist (see, e.g., the states 0 and 3 of Table 3 ). The excess rate can be considered as a measure of the bit stream redundancy.

\section{ESTIMATION ALGORITHM}

The above models of dependencies can be exploited to help the estimation of the bit stream (hence of the symbol sequence). The MAP estimation criterion corresponds to the optimal Bayesian estimation of a process $X$, given available measurements $Y$ :

$$
\hat{X}=\arg \max _{x} \mathbb{P}(X=x \mid Y) .
$$

However, if the mean square error (MSE) is the performance measure, the MAP criterion is suboptimal. The conditional mean or minimum MSE (MMSE) is in this case the optimal decoder. The decoder then seeks a sequence of symbol reproductions that will minimize the expected distortion, given the sequence of observations denoted by $E[D(\hat{A}, A) \mid Y]$. These expected distortions can be computed in a very straightforward way, given the MAP estimates, provided the probability measures on the sequence of binary symbols $S$ are converted into probability measures on the sequence of $M$-ary symbols $A$. In Section 10, only the MAP estimates have been considered.

The optimization is performed over all possible sequences $x$. This applies directly to the estimation of the hidden states of the processes $(X, N)$ (symbol-clock model of source + coder) and $(X, K)$ (bit-clock model of source + decoder), given the sequence of measurements. The estimation is run on the source-decoder product model in order to avoid handling the $n \mathrm{scl}_{k}$ variable.

Estimating the set of hidden states $(X, K)=\left(X_{1}, K_{1}\right) \cdots$ $\left(X_{N}, K_{N}\right)$ is equivalent to estimating the associated sequence of decoded symbols $S=S_{1} \cdots S_{K_{n}} \cdots S_{K_{N}}$, given the measurements $Y_{1}^{N}$ at the output of the channel. The best sequence $(X, K)$ can be obtained from the local probabilities on the pairs $\left(X_{n}, K_{n}\right)$ by the equation

$$
\mathbb{P}(X, K \mid Y)=\prod_{n=1}^{N} \mathbb{P}\left(X_{n}, K_{n} \mid Y\right) .
$$

The computation of the entity $\mathbb{P}\left(X_{n}, K_{n} \mid Y\right)$ can be organized around the factorization

$$
\mathbb{P}\left(X_{n}, K_{n} \mid Y\right) \propto \mathbb{P}\left(X_{n}, K_{n} \mid Y_{1}^{n}\right) \cdot \mathbb{P}\left(Y_{n+1}^{N} \mid X_{n}, K_{n}\right)
$$


where $\propto$ denotes a renormalization factor. The Markov property allows a recursive computation of both terms of the right-hand side, using the BCJR algorithm [21]. The forward sweep concerns the first term

$$
\begin{aligned}
\mathbb{P}\left(X_{n}=x_{n}, K_{n}=k_{n} \mid Y_{1}^{n}\right) & \\
=\sum_{\left(x_{n-1}, k_{n-1}\right)} & \mathbb{P}\left(X_{n-1}=x_{n-1}, K_{n-1}=k_{n-1} \mid Y_{1}^{n-1}\right) \\
\cdot & \mathbb{P}\left(X_{n}=x_{n}, K_{n}=k_{n} \mid X_{n-1}=x_{n-1}, K_{n-1}=k_{n-1}\right) \\
\cdot & \mathbb{P}\left(U_{n}=u_{\left(x_{n-1}, k_{n-1}\right)\left(x_{n}, k_{n}\right)} \mid Y_{n}\right) .
\end{aligned}
$$

The terms on the right-hand side of the equation are, respectively, the recursive term, the transition probability given by the product model (see Section 6), and the probability to have emitted the bit $U_{n}$ triggering the transition between $X_{n-1}=x_{n-1}$ and $X_{n}=x_{n}$, given the measure $Y_{n}$ (channel model). The process is initialized at the starting state $(0,0)$ and allows to compute $\mathbb{P}\left(X_{n}, K_{n} \mid Y_{1}^{n}\right)$ for all possible states $\left(x_{n}, k_{n}\right)$ and for each bit-clock instant $n=1, \ldots, N$.

The backward sweep provides the second term in (10):

$$
\begin{aligned}
\mathbb{P}\left(Y_{n+1}^{N} \mid X_{n}=x_{n}, K_{n}=k_{n}\right) & \\
=\sum_{\left(x_{n+1}, k_{n+1}\right)} & \mathbb{P}\left(Y_{n+2}^{N} \mid X_{n+1}=x_{n+1}, K_{n+1}=k_{n+1}\right) \\
\cdot & \mathbb{P}\left(X_{n+1}=x_{n+1}, K_{n+1}=k_{n+1} \mid X_{n}=x_{n}, K_{n}=k_{n}\right) \\
\cdot & \mathbb{P}\left(U_{n+1}=u_{\left(x_{n}, k_{n}\right)\left(x_{n+1}, k_{n+1}\right)} \mid Y_{n+1}\right) .
\end{aligned}
$$

The process is initialized for all possible "last" states $\left(x_{N}, k_{N}\right)$ and allows to compute $\mathbb{P}\left(Y_{n+1}^{N} \mid X_{n}, K_{n}\right)$ for all possible states $\left(x_{n}, k_{n}\right)$ and for each bit-clock instant consecutively from $N$ to 1 .

As in $[2,5]$, a termination constraint can be introduced; one can ensure that the decoder produces the right number of symbols $\left(K_{N}=K\right)$ (if known). All the paths in the trellis which do not lead to a valid sequence length are suppressed. The trellis on which the estimation is performed can be precomputed, with all transitions and outputs stored. Figure 9 shows the trellis computed with the example product model of Figure 7 for a sequence of $K=7$ symbols producing $N=$ 6 bits.

\section{SOFT SYNCHRONIZATION}

Termination constraints mentioned in Section 7 can be regarded as means to force synchronization at the end of the sequence; they indeed constrain the decoder to have the right number of symbols $\left(K_{N}=K\right)$ (if known) after decoding the estimated bit stream $\hat{U}$. These constraints ensure synchronization at the end of the bit stream, but do not ensure synchronization in the middle of the sequence. One can introduce extra information specifically to help the resynchronization "in the middle" of the sequence. For this, we consider here the introduction of extra bits at some known po- sitions $I_{s}=\left\{i_{1}, \ldots, i_{s}\right\}$ in the symbol stream. This extra information takes the form of dummy binary symbols (in the spirit of the techniques described in $[11,12,14,17,18])$ inserted in the binary symbol stream at some known symbolclock positions after the conversion of the $M$-ary source into the binary source. Since these dummy symbols are inserted at some known symbol-clock instants, the position of the corresponding extra bits in the coded bit stream depends on the sequence of symbols encoded, hence is random.

Models and algorithms above have to account for this extra information. Inserting an extra dummy symbol at known positions in the symbol stream amounts to add a section with deterministic transitions in the binary tree model of the source. The presence of this extra information can be exploited by the estimation. During the estimation process, the variable $K_{n}$ indicates when a marker should be expected. The corresponding transition probabilities in the estimation trellis are updated accordingly. A null probability is given to all transitions that do not emit the expected sequence of binary symbols, while a probability of one is set to the others. Therefore, some paths in the estimation trellis become forbidden and can be suppressed, leading to a reduction of the number of states.

\section{ITERATIVE CC-AC DECODING ALGORITHM}

The soft synchronization mechanism described above increases significantly the reliability of the segmentation and estimation of the sequence of symbols. One can however consider, in addition, the usage of an error correction code, for example, of a systematic convolutional CC. Both codes can be concatenated in the spirit of serial turbo codes. Adopting this principle, one can therefore work on each model (quasi-arithmetic coder and channel coder) separately and design an iterative estimator, provided an interleaver is introduced between the models. The structure of dependencies between the variables involved is outlined in Figure 10.

Such a scheme requires extrinsic information on the bits $U_{n}$ to be transmitted by the CC to the soft arithmetic decoder and reciprocally. The extrinsic information on a bit $U_{n}$ represents the modification induced by the introduction of the rest of the observations $Y_{1}^{n-1}, Y_{n+1}^{N}$ on the conditional law of $U_{n}$ given $Y_{n}$. The extrinsic information can be expressed as

$$
\operatorname{Ext}_{U_{n}}\left(Y \mid Y_{n}\right) \propto \frac{\mathbb{P}\left(U_{n} \mid Y\right)}{\mathbb{P}\left(U_{n} \mid Y_{n}\right)} .
$$

The iterative estimation proceeds by first running a BCJR algorithm on the channel coder model. The extrinsic information on the useful bits $U_{n}$ is a direct subproduct of the BCJR algorithm. These measurements can in turn be used as input for the estimation run on the quasi-arithmetic decoder model described above.

The result of the quasi-arithmetic soft decoding procedure is the a posteriori probabilities on the states $\left(X_{n}, K_{n}\right)$ of the estimation trellis, given the observations $\mathbb{P}\left(X_{n}, K_{n} \mid Y\right)$. These a posteriori probabilities must then be converted into extrinsic information on bits $U_{n}$. The probability of having a 


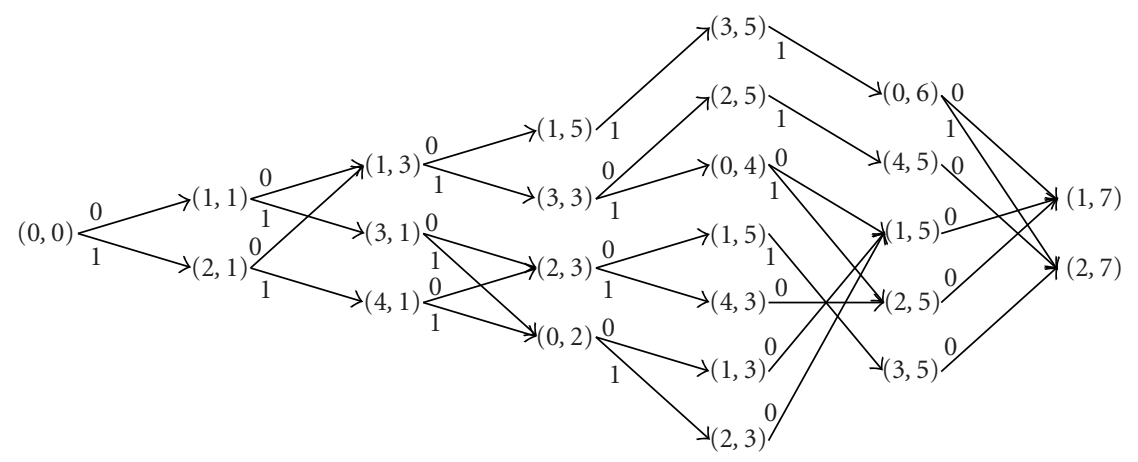

FIgURE 9: Example of trellis for $K=7$ symbols and $N=6$ bits.

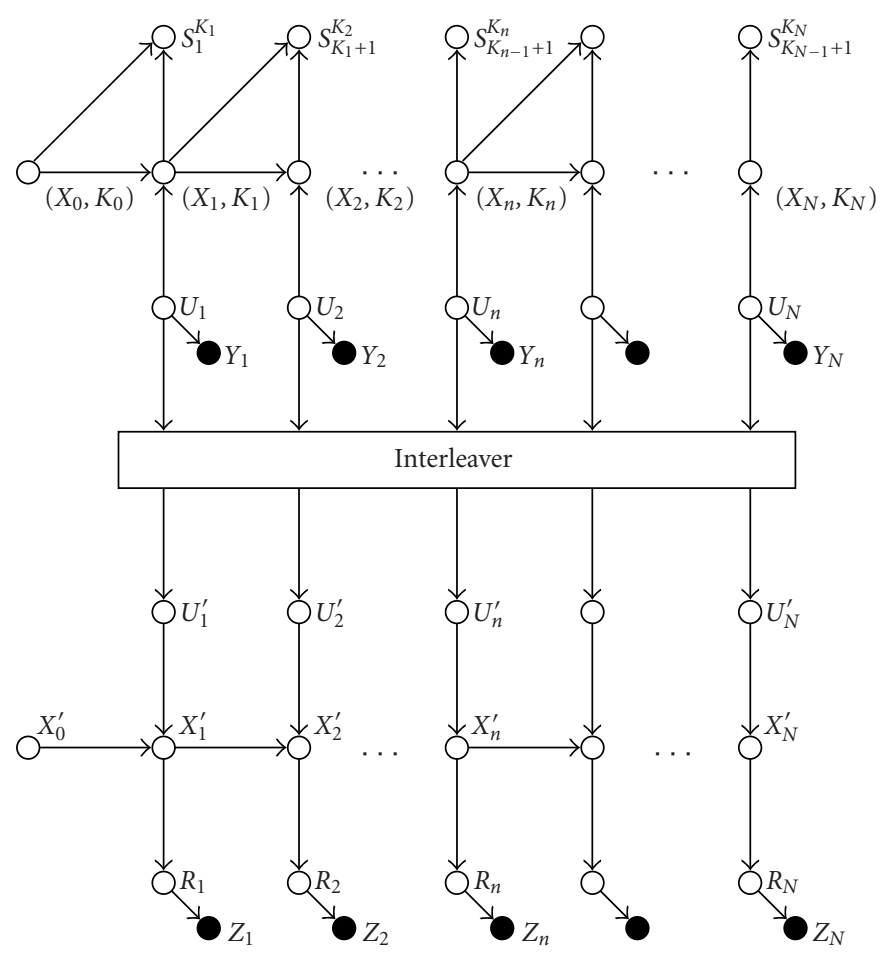

FIGURE 10: Graphical representation of dependencies in the joint arithmetic-channel coding chain.

bit $U_{n}=u_{n}$ given $Y$ is the sum of the transition probabilities between all states $\left(x_{n-1}, k_{n-1}\right)$ and $\left(x_{n}, k_{n}\right)$ for which this transition exists and is triggered by $u_{n}$. Thus, the a posteriori probability of a bit $U_{n}$ given the observations is obtained by the equation

$$
\begin{aligned}
& \left.P\left(U_{n}=u_{n} \mid Y\right)\right|_{u_{n}=0,1} \\
& =\sum_{\left(x_{n-1}, k_{n-1}\right)} \mathbb{P}\left(x_{n-1}, k_{n-1} \mid Y\right) \\
& \cdot \frac{\mathbb{P}\left(\operatorname{succ}_{u_{n}}\left(x_{n-1}, k_{n-1}\right) \mid Y\right)}{\sum_{u_{n}=0,1} \mathbb{P}\left(\operatorname{succ}_{u_{n}}\left(x_{n-1}, k_{n-1} \mid Y\right)\right)},
\end{aligned}
$$

where $\operatorname{succ}_{u_{n}}\left(x_{n-1}, k_{n-1}\right)$ is the state $\left(x_{n}, k_{n}\right)$ reached by the transition from $\left(x_{n-1}, k_{n-1}\right)$ triggered by the bit $U_{n}=u_{n}$.

\section{EXPERIMENTAL RESULTS}

To evaluate the performance of the soft quasi-arithmetic decoding procedure, a set of experiments has been performed on a first-order Gauss-Markov source, with zeromean, unit-variance, and different correlation factors $\rho$. The source is quantized with an eight-level uniform quantizer (3 bits) on the interval $[-3,3]$. We consider sequences of $K=200$ symbols with different source correlation factors. All the simulations have been performed assuming an additive white Gaussian channel with a binary phase shift keying (BPSK) modulation. The results are averaged over 3000 realizations.

The first experiment aimed at comparing the performances in terms of soft decoding of Huffman codes [7], 
arithmetic codes [19], and quasi-arithmetic codes with $T=4$ for comparable overall rates. When the source correlation increases, the compression efficiency of the arithmetic coder increases; soft synchronization patterns are inserted in the arithmetically encoded stream up to a comparable overall rate. Figures 11 and 12 show the residual SERs and SNR obtained for different channel $E_{b} / N_{0}$ for, respectively, $\rho=0.5$ and $\rho=0$.9. For sources with low correlation $(\rho=0.5$ and under) and for values of $E_{b} / N_{0}$ lower than $5 \mathrm{~dB}$ (i.e., for high bit error rates), quasi-arithmetic coding outperforms both arithmetic and Huffman coding. However, for sources with high correlation, the quasi-arithmetic coder and decoder turn out to be slightly less efficient than the optimal arithmetic coder for this range of $E_{b} / N_{0}$. The reason is that excess rate induced by quasi-arithmetic coding increases with the source correlation (see Section 4.3). The optimal arithmetic coding fully exploiting the source correlation, one can then insert a higher amount of soft synchronization patterns, for the same overall rate, resulting in an improved error resilience. The same trend has been observed for different rates. The gain brought on soft quasi-arithmetic decoding by synchronization markers is illustrated in Figures 13 and 14, respectively, for $\rho=0.5$ and $\rho=0.9$. Notice that, even for high correlation sources, the performances of the quasi-arithmetic decoder would obviously increase if one would allow a higher complexity, that is, a higher value for the parameter $T$. In order to compare fairly the three methods, one must also consider their complexity. It can be measured by the size of the trellis used for the estimation. Considering a sequence of 200 symbols, soft decoding of Huffman codes needs two trellises with about 900 states, respectively, per bit-clock and symbol-clock instants. The complexity of soft decoding of arithmetic codes is limited to 100 states per symbol-clock instant, using pruning. Finally, the soft decoding of quasi-arithmetic codes leads to a trellis containing about 2400 states per bit-clock instant, hence being the most complex of the three. Pruning may be considered to reduce this complexity.

The second experiment aimed at evaluating the performance of the iterative channel/quasi-arithmetic decoding algorithm. Figures 15 and 16 depict the SER and SNR performances in comparison with the decoding approach with soft synchronization, respectively, for $\rho=0.5$ and $\rho=0.9$. The first observation is that the iterations bring significant improvements and higher gain is being obtained when the source correlation is high (see Figure 16). Nevertheless, if the source correlation is low, the soft synchronization outperforms the iterative scheme. In this range of channel SNRs, the CC cannot correct all the errors, and the desynchronization phenomenon prevails. It is therefore preferable to dedicate redundancy within the source to fight against these desynchronizations. In contrast, when the source correlation is high $(\rho=0.9)$, the SER is lower with the iterative solution due to a proper exploitation of the intersymbol correlation for segmenting and estimating the bit stream. It can also be observed that the gain brought by the iterations depends on the degree of redundancy present on both sides of the interleaver. Figures 17 and 18 show the performances obtained when combining synchronization markers and channel coding, respectively, for $\rho=0.5$ and $\rho=0.9$. Three approaches are compared, with equal or sufficiently close overall rates. In the first one, only channel coding $(k / n=2 / 3$ and 4 iterations) is used to add redundancy. In the second one, channel coding ( $k / n=5 / 6$ and 4 iterations) is combined with synchronization markers. Finally, in the third approach, only synchronization markers are used. For sources with high correlation, channel coding leads to higher performance since the correlation present in the source is already exploited efficiently to fight against desynchronizations. For sources with low correlation and for values of $E_{b} / N_{0}$ lower than $3 \mathrm{~dB}$, on the contrary, the combination of channel coding and synchronization markers has been found to be the best strategy. It has also been observed that, in this case, iterations bring a higher gain than in other cases.

In another set of experiments, the influence of the length of the sequence on the system performance is considered. Figures 19 and 20 depict the SER and SNR performances with three different lengths, respectively, for $\rho=0.5$ and $\rho=0.9$. As expected, the performance decreases when the length of the sequence increases. This can be explained by the exploitation of the termination constraint which contributes to the decoder resynchronization. The length of the sequence has also an influence on the complexity of the decoding. Indeed, this complexity depends on the size of the estimated trellis, which depends mainly on the excursion, that is, the possible values, of the variable $K_{n}$. The excursion is higher in the middle than at the extremities of the sequence. Hence, it reaches higher values with longer sequences. We have measured experimentally the average number of states of the trellis per bit-clock instant $n$. The values obtained are 557,1177 , and 2376, respectively, for sequences of 50, 100, and 200 symbols. Pruning techniques may be required for longer sequences.

In the last experiment, the impact of the precision of the quasi-arithmetic coder parameterized by the variable $T$ has been analyzed. Figure 21 provides the SER and SNR performances for $T=4$ and $T=8$ without extra redundancy, and for $T=8$ with soft synchronization patterns added to obtain a bit rate comparable to the case where $T=4(\rho=0.5)$. The lower precision coder $(T=4)$ due to the presence of residual redundancy is more resilient to errors. However, the coder with a higher precision $(T=8)$ allows, by better exploiting the source statistics, to obtain higher compression efficiency and in turn dedicate redundancy to resynchronize the decoding process. Then the overall performances appear to be similar for $E_{b} / N_{0}$ larger than $4 \mathrm{~dB}$. Once again, the complexity is an issue. Indeed, increasing the value of $T$ leads necessarily to a higher number of states. In this experiment, we have found the following average number of states of the estimated trellis per bit-clock instant: 1193 when $T=4,4736$ when $T=8$, and 4297 when $T=8$ with the insertion of synchronization markers. Therefore, it is highly desirable to choose $T$ as small as possible. 


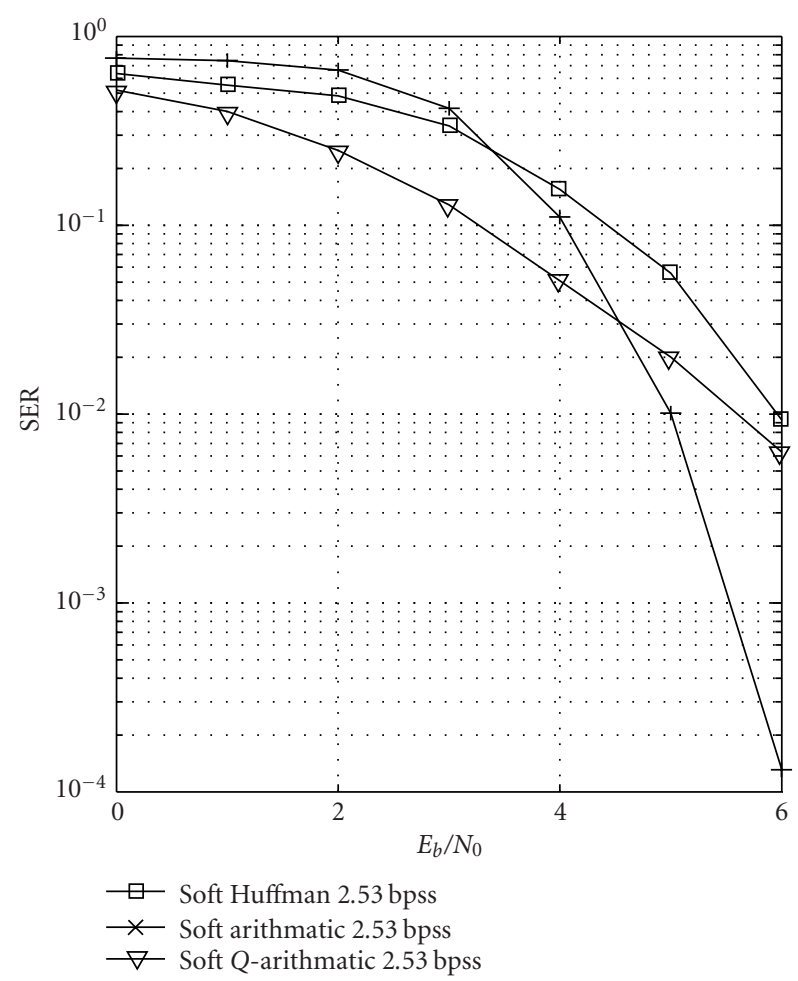

(a)

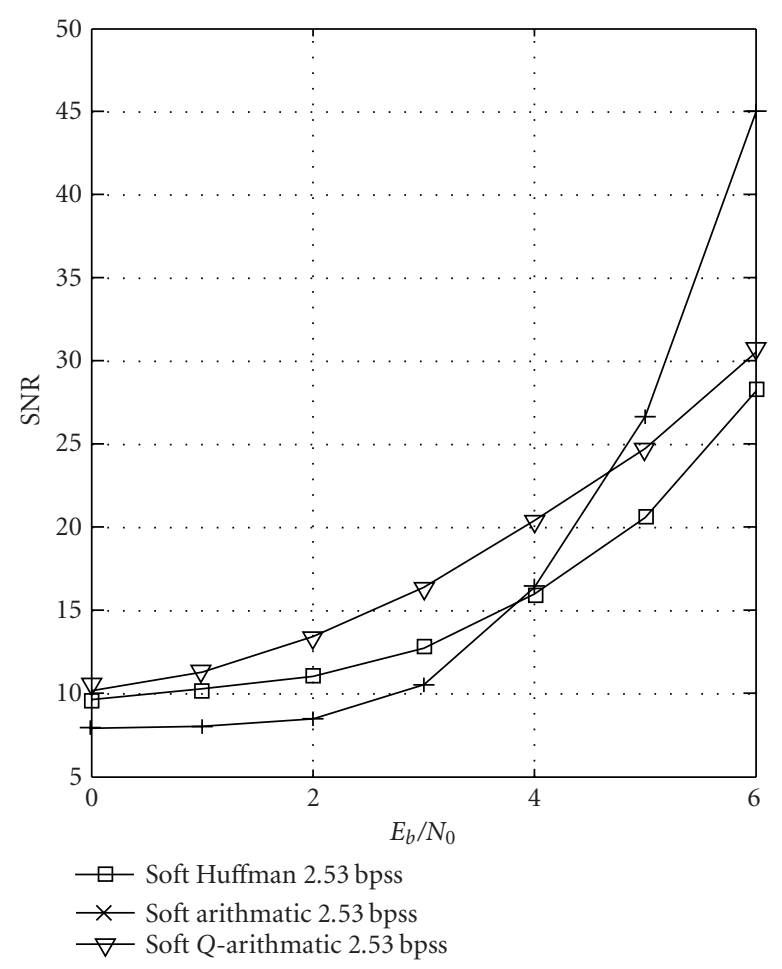

(b)

FIGURE 11: SER and SNR performances of (i) soft Huffman decoding, (ii) soft arithmetic decoding, and (iii) soft quasi-arithmetic decoding ( $\rho=0.5,200$ symbols, 3000 channel realizations).

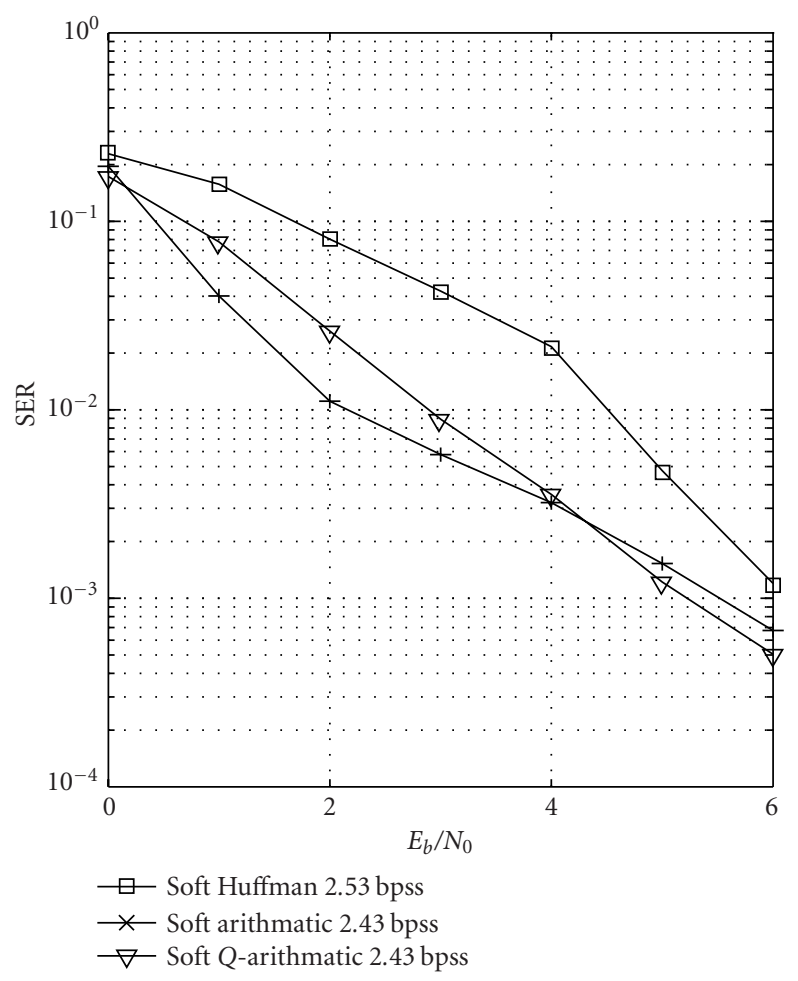

(a)

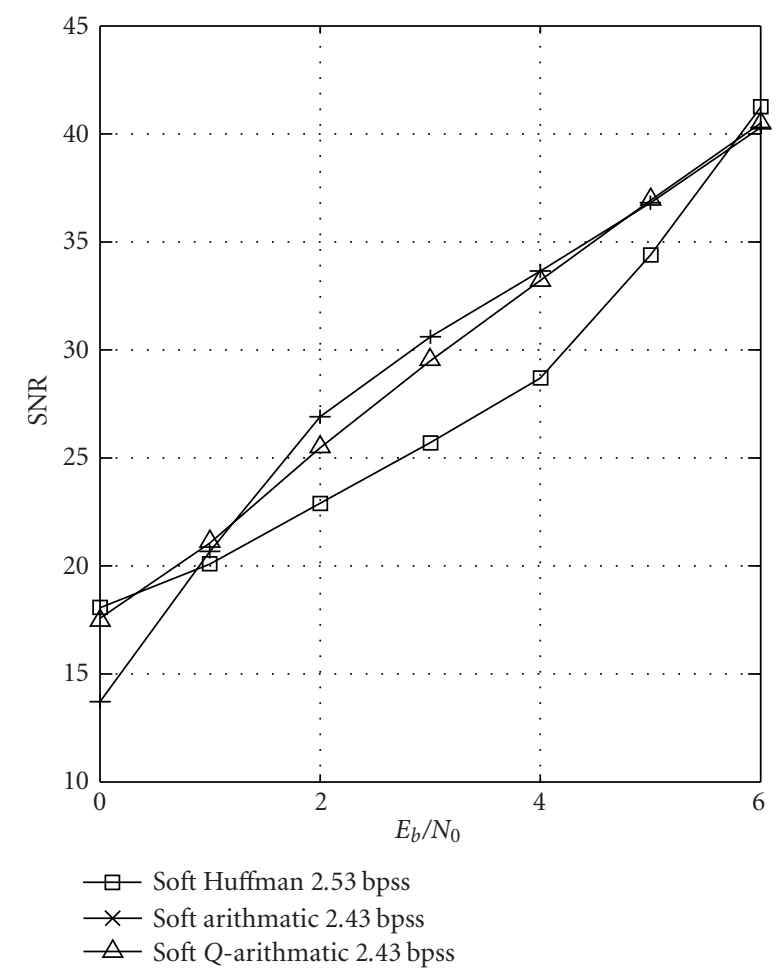

(b)

FIGURE 12: SER and SNR performances of (i) soft Huffman decoding, (ii) soft arithmetic decoding, and (iii) soft quasi-arithmetic decoding ( $\rho=0.9,200$ symbols, 3000 channel realizations). 


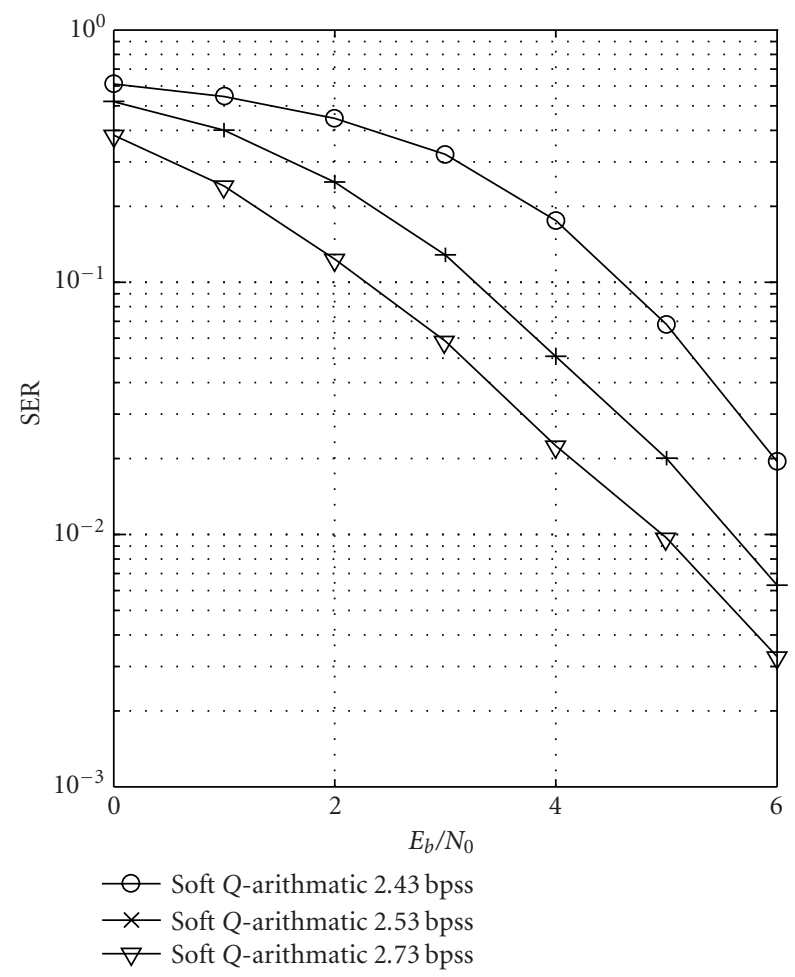

(a)

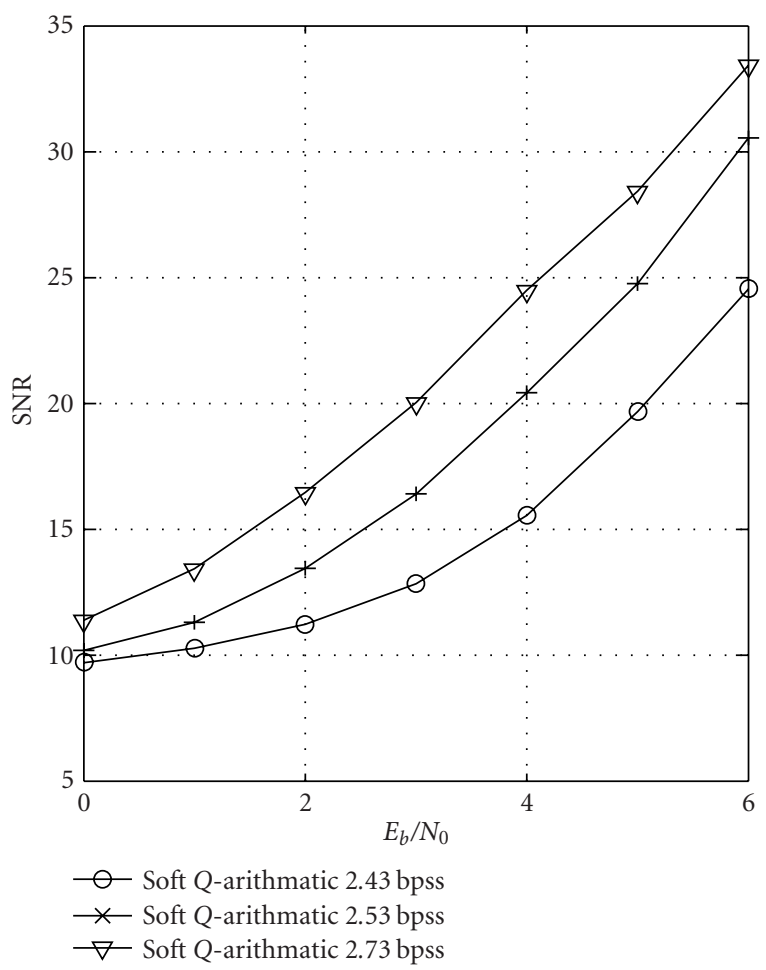

(b)

FIGURE 13: SER and SNR performances of soft quasi-arithmetic decoding for different rates ( $\rho=0.5,200$ symbols, 3000 channel realizations).

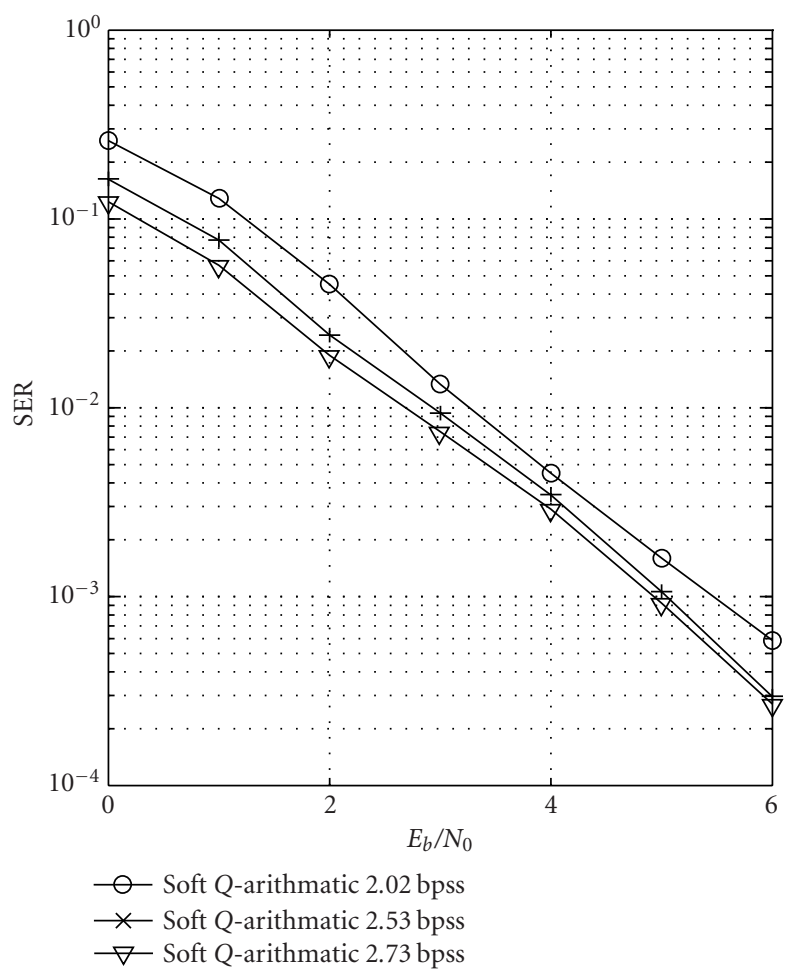

(a)

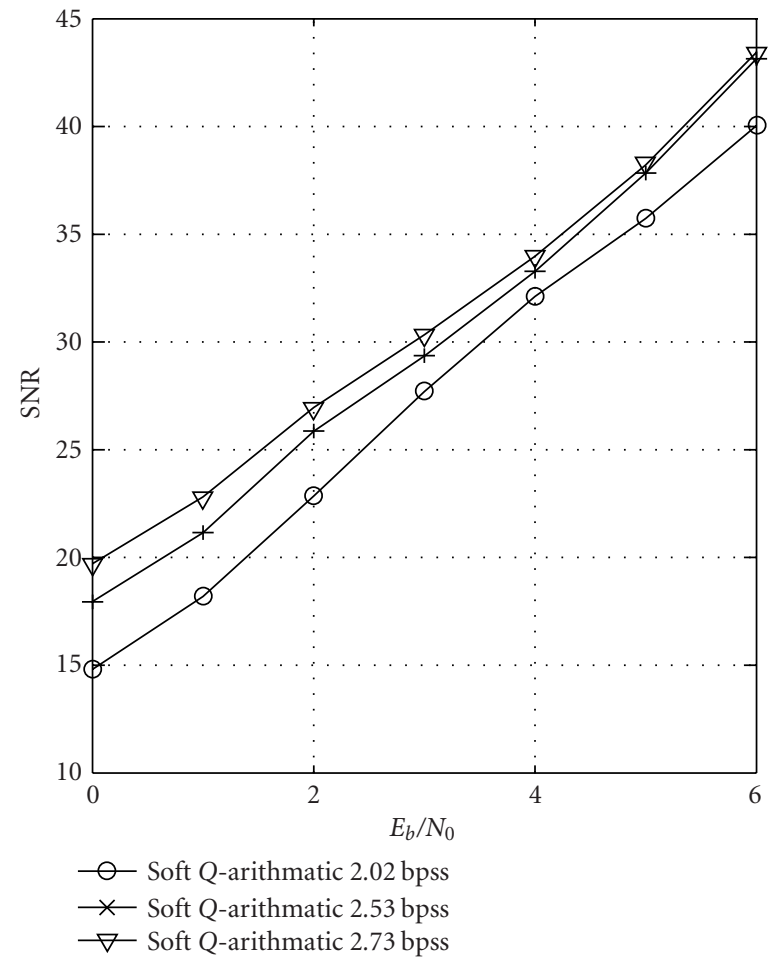

(b)

FIGURE 14: SER and SNR performances of soft quasi-arithmetic decoding for different rates ( $\rho=0.9,200$ symbols, 3000 channel realizations). 


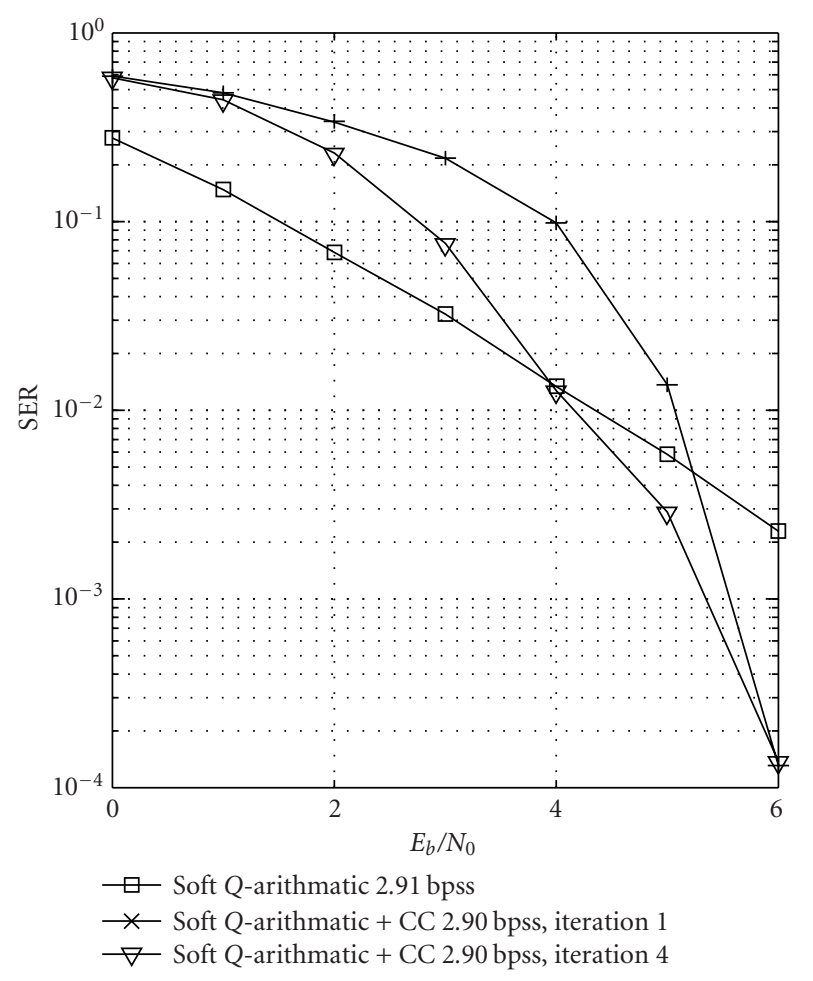

(a)

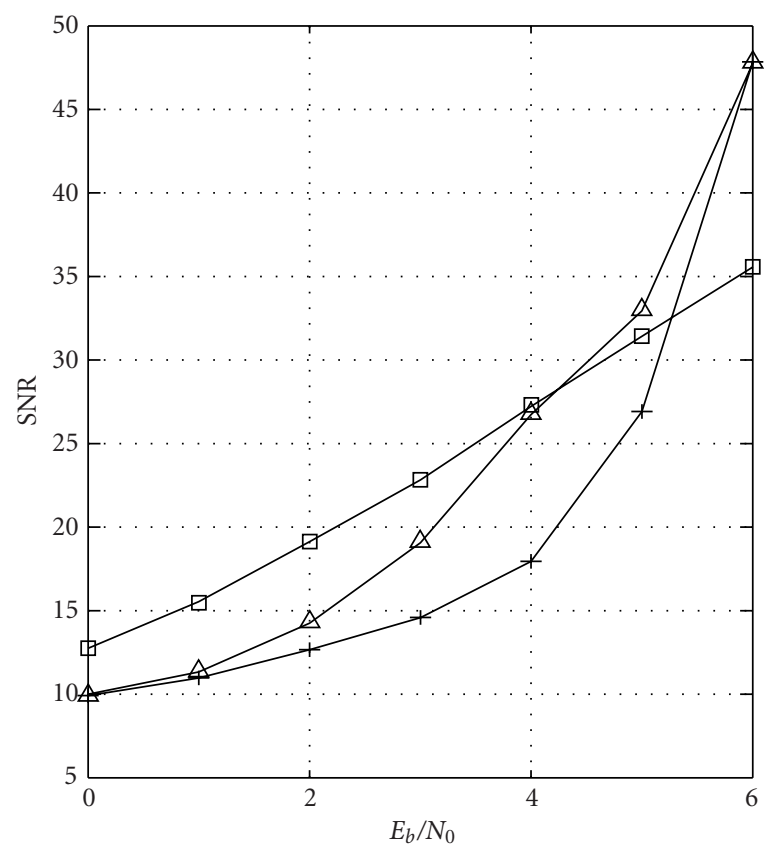

$\square$ Soft $Q$-arithmatic 2.91 bpss
$\times$ Soft $Q$-arithmatic + CC 2.90 bpss, iteration 1

$\triangle$ Soft Q-arithmatic + CC 2.90 bpss, iteration 4

(b)

FIGURE 15: SER and SNR performances of (i) soft quasi-arithmetic decoding with soft synchronization and (ii) turbo quasi-arithmetic/ channel decoding ( $\rho=0.5,200$ symbols, 3000 channel realizations).

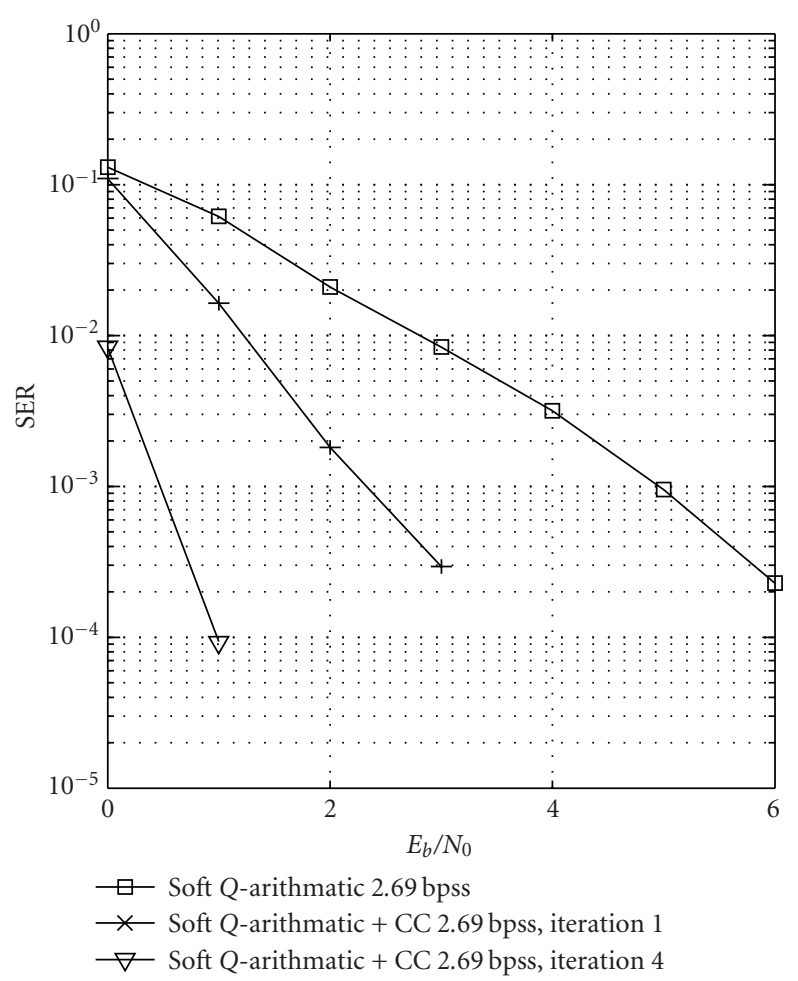

(a)

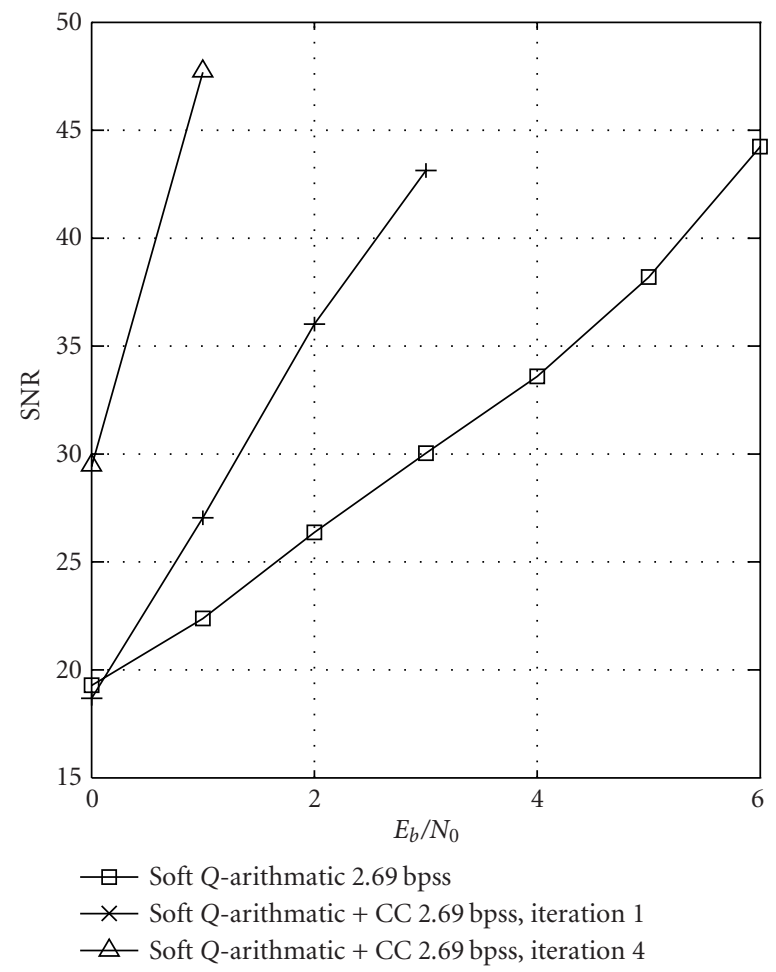

(b)

FIGURE 16: SER and SNR performances of (i) soft quasi-arithmetic decoding with soft synchronization and (ii) turbo quasi-arithmetic/ channel decoding ( $\rho=0.9,200$ symbols, 3000 channel realizations). 


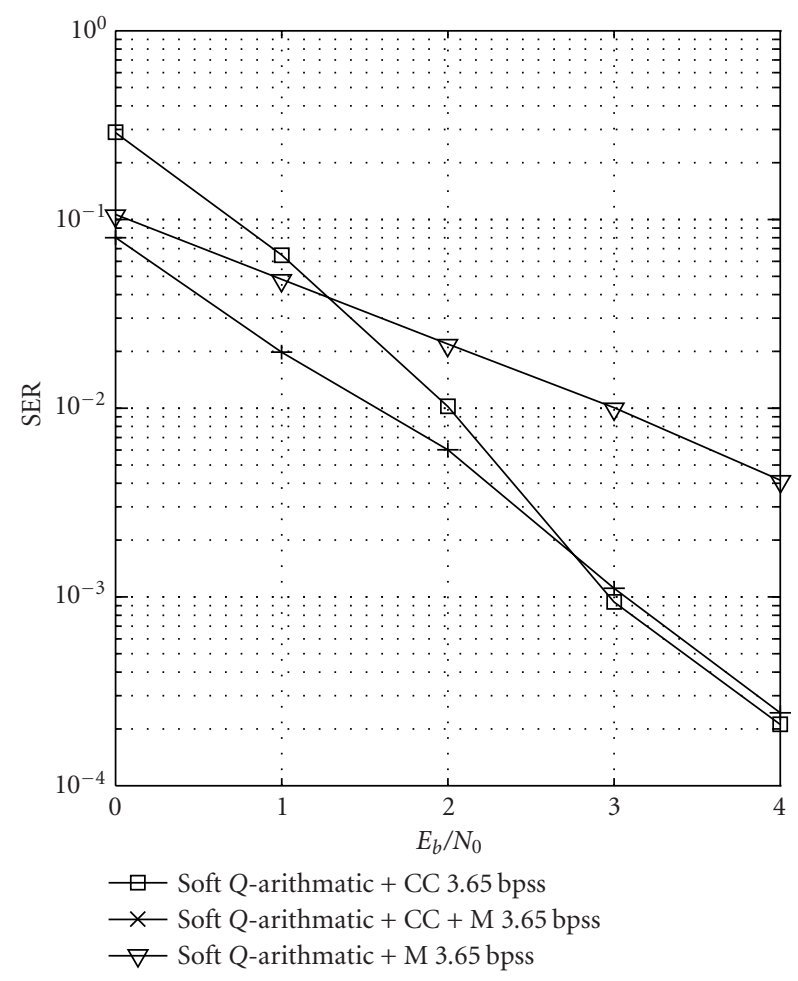

(a)

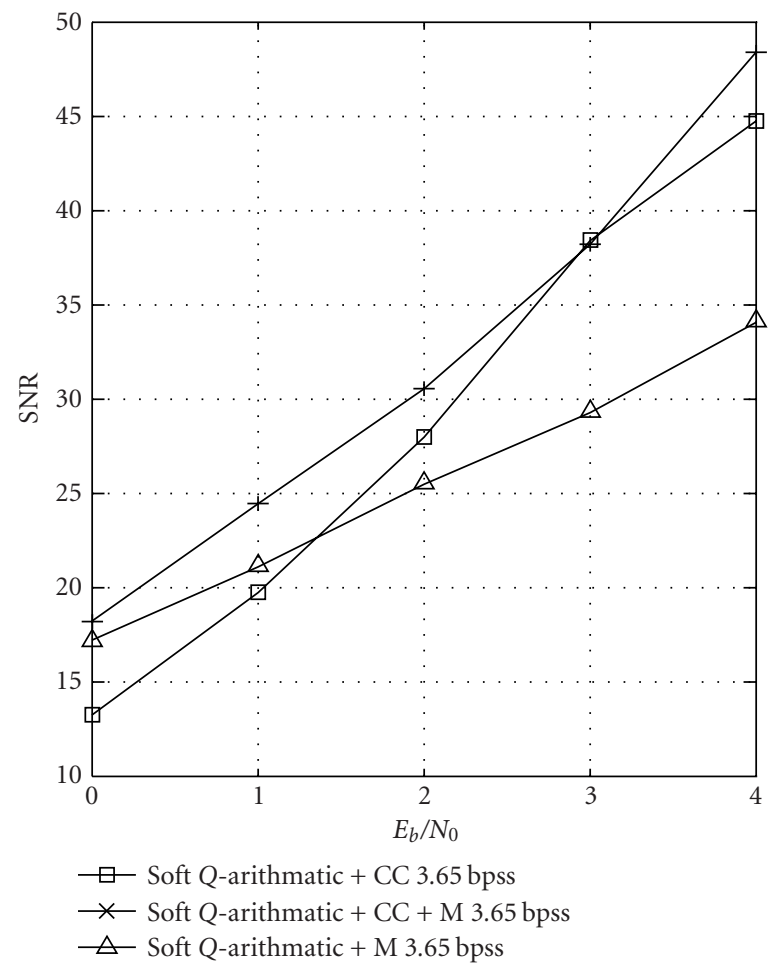

(b)

FIGURE 17: SER and SNR performances of (i) turbo quasiarithmetic/channel decoding, (ii) turbo quasi-arithmetic/channel decoding with soft synchronization, and (iii) soft quasi-arithmetic decoding with soft synchronization ( $\rho=0.5,200$ symbols, 1500 channel realizations).

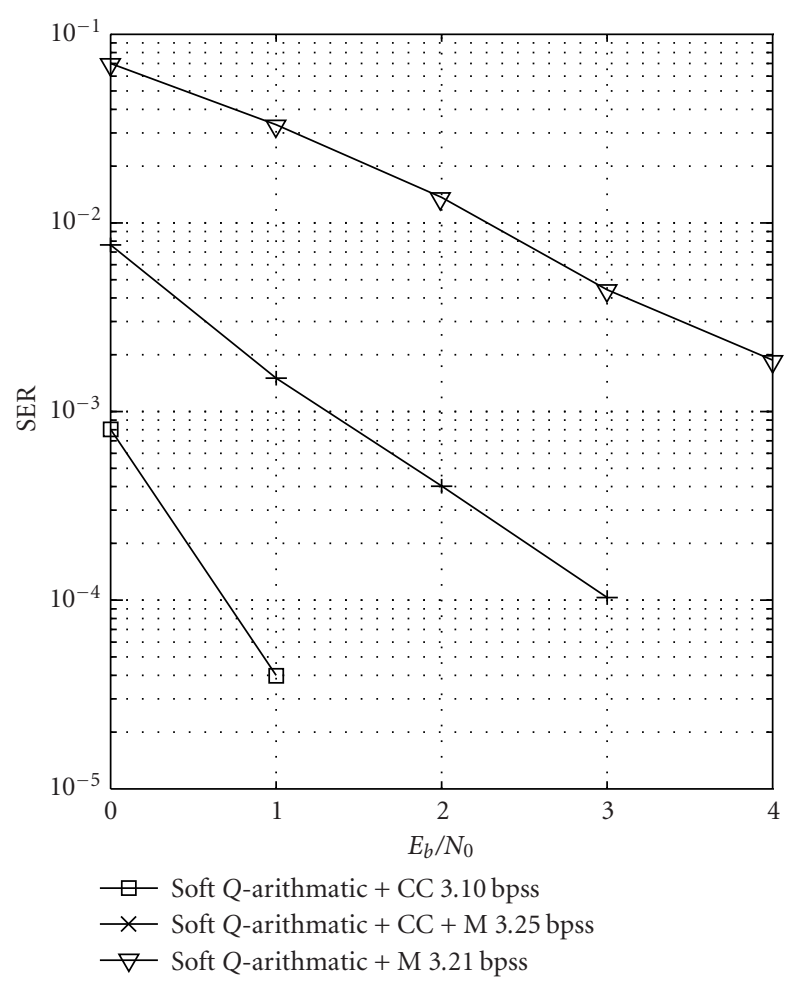

(a)

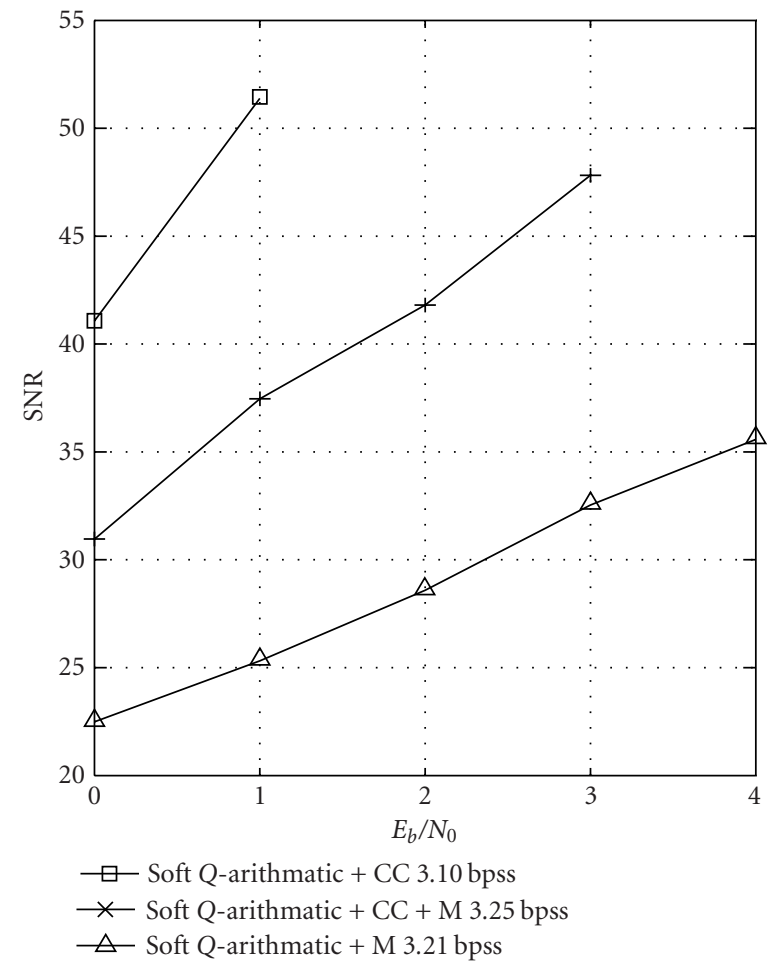

(b)

FIGURE 18: SER and SNR performances of (i) turbo quasiarithmetic/channel decoding, (ii) turbo quasi-arithmetic/channel decoding with soft synchronization, and (iii) soft quasi-arithmetic decoding with soft synchronization $(\rho=0.9,200$ symbols, 1500 channel realizations). 


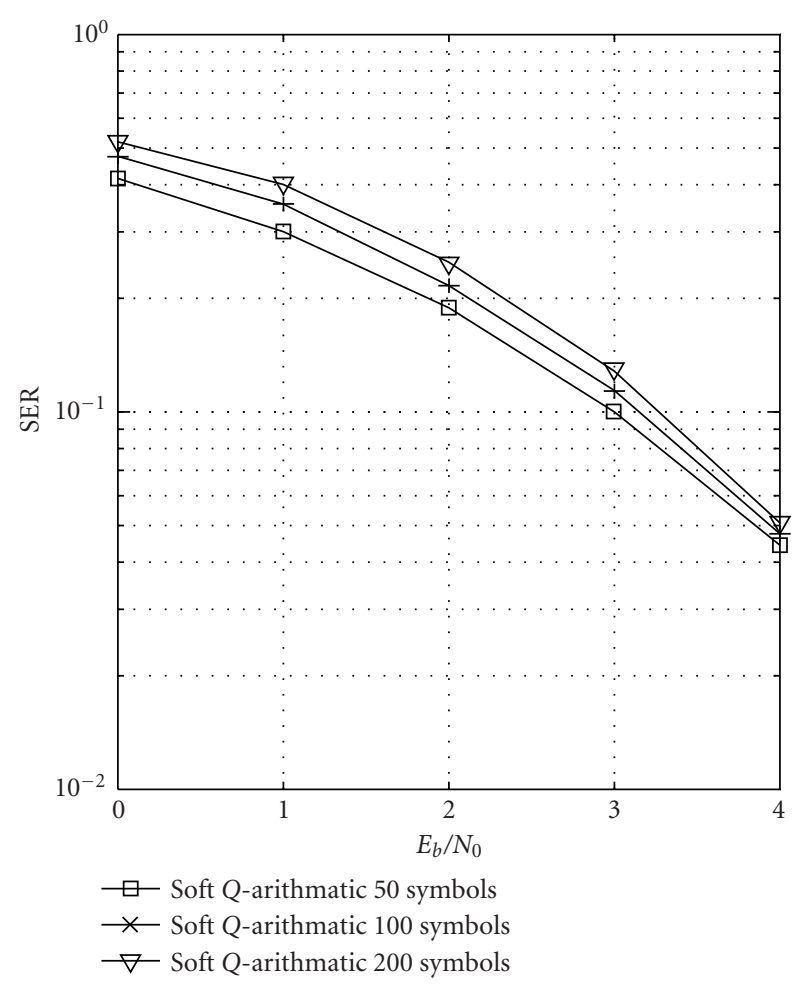

(a)

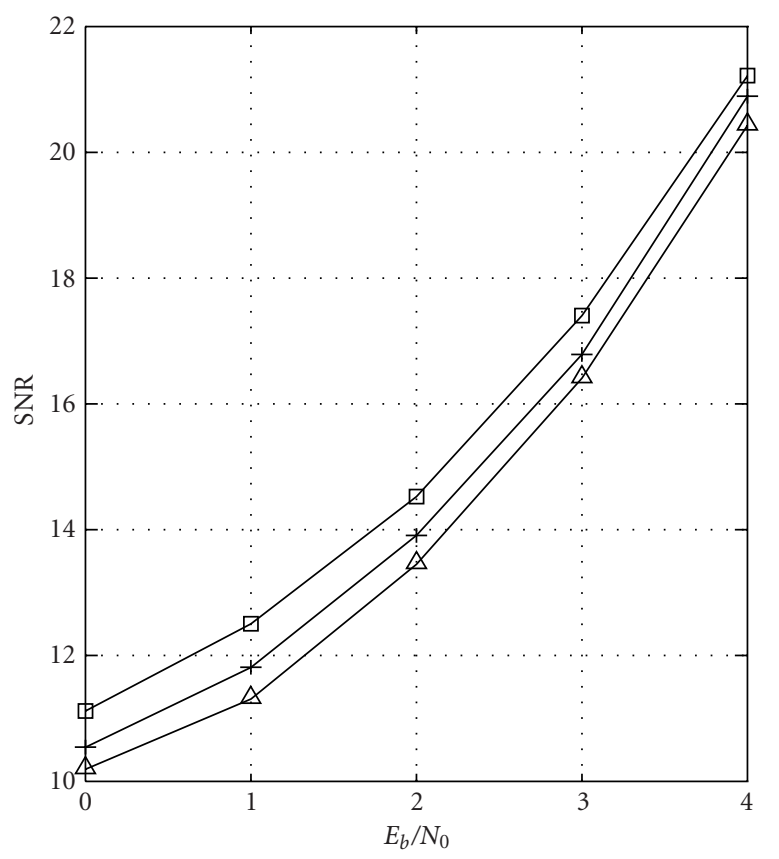

—-Soft $Q$-arithmatic 50 symbols

$\times$ Soft $Q$-arithmatic 100 symbols

$\triangle$ Soft Q-arithmatic 200 symbols

(b)

FIGURE 19: SER and SNR performances of soft quasi-arithmetic decoding for, respectively, 50, 100, and 200 symbols ( $\rho=0.5,3000$ channel realizations).

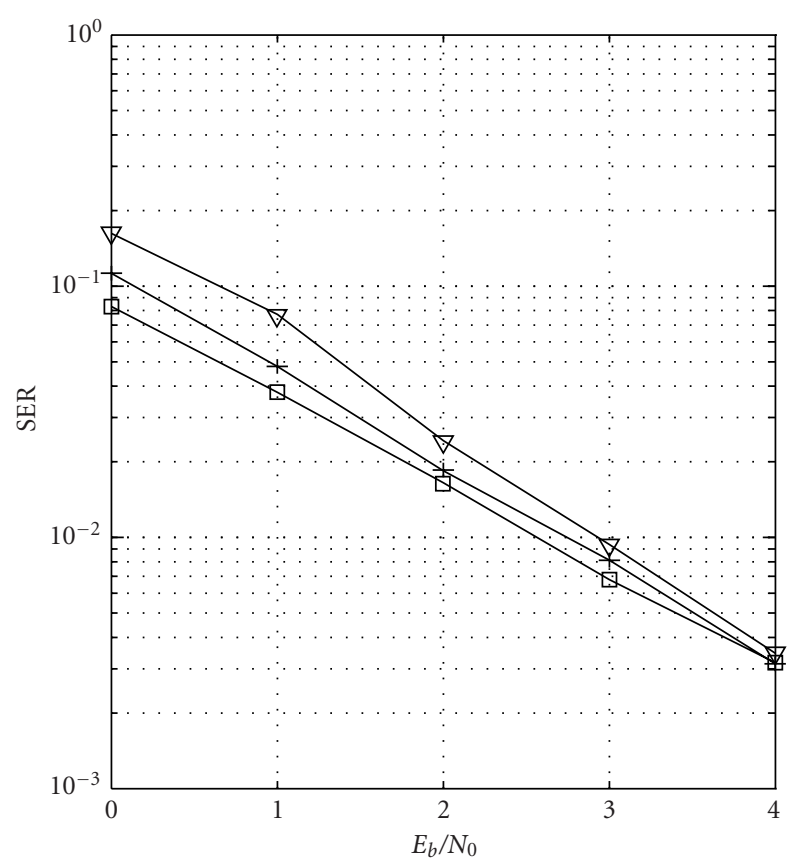

$\square$ Soft $Q$-arithmatic 50 symbols
$¥$ Soft $Q$-arithmatic 100 symbols
$\nabla$ Soft Q-arithmatic 200 symbols

(a)

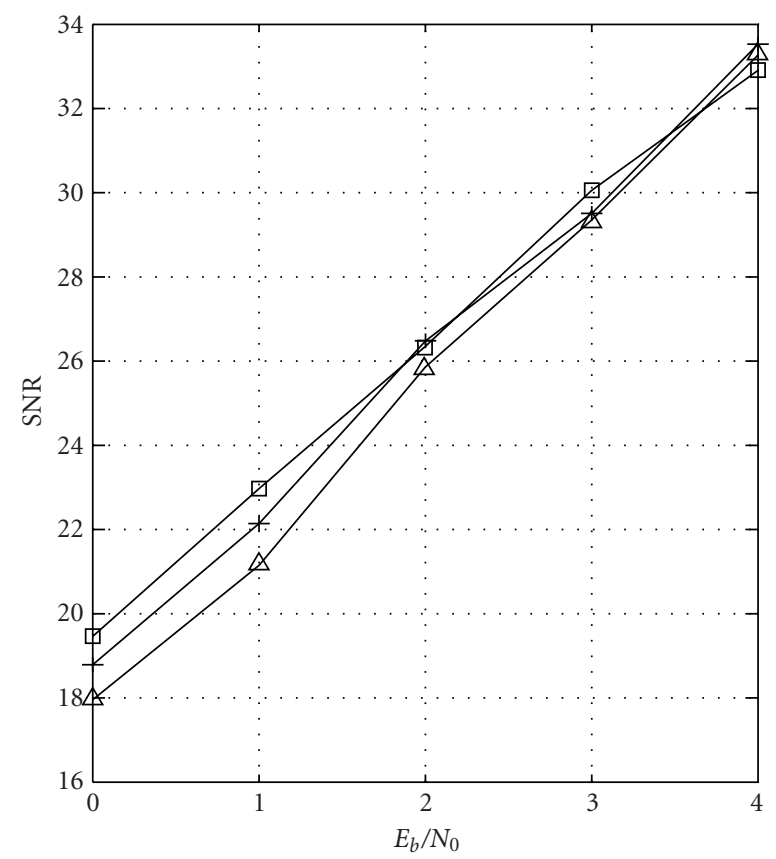

$\square$ Soft Q-arithmatic 50 symbols

$\leftarrow$ Soft $Q$-arithmatic 100 symbols

$\triangle$ Soft Q-arithmatic 200 symbols

(b)

FIGURE 20: SER and SNR performances of soft quasi-arithmetic decoding for, respectively, 50, 100, and 200 symbols ( $\rho=0.9,3000$ channel realizations). 


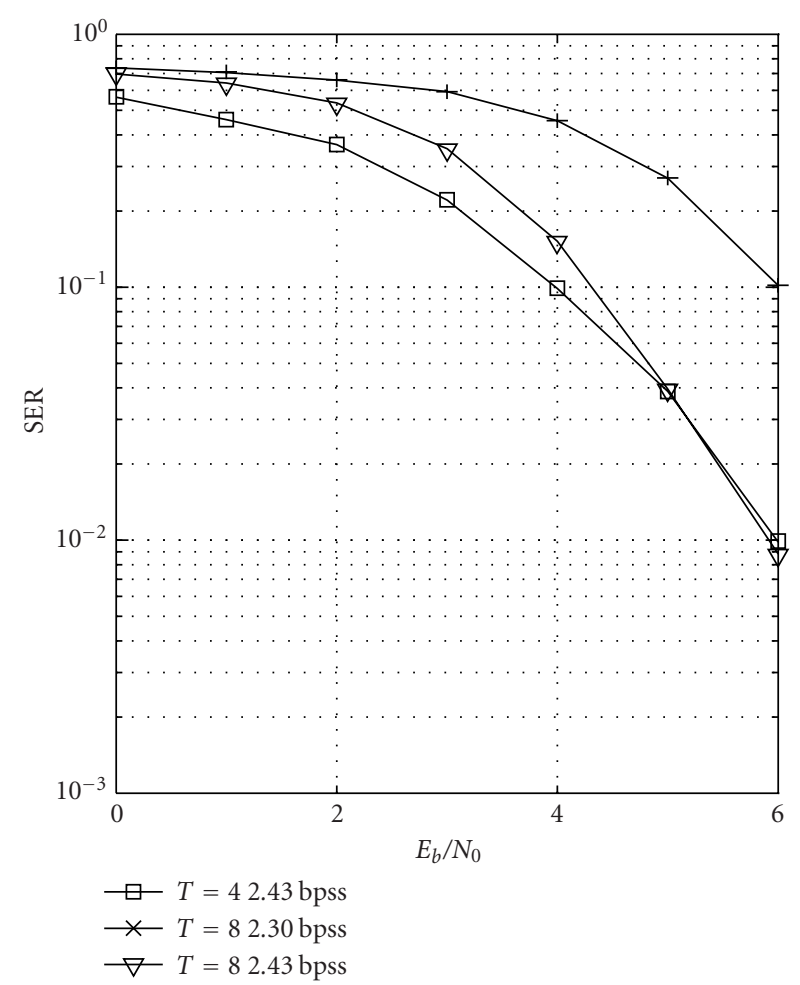

(a)

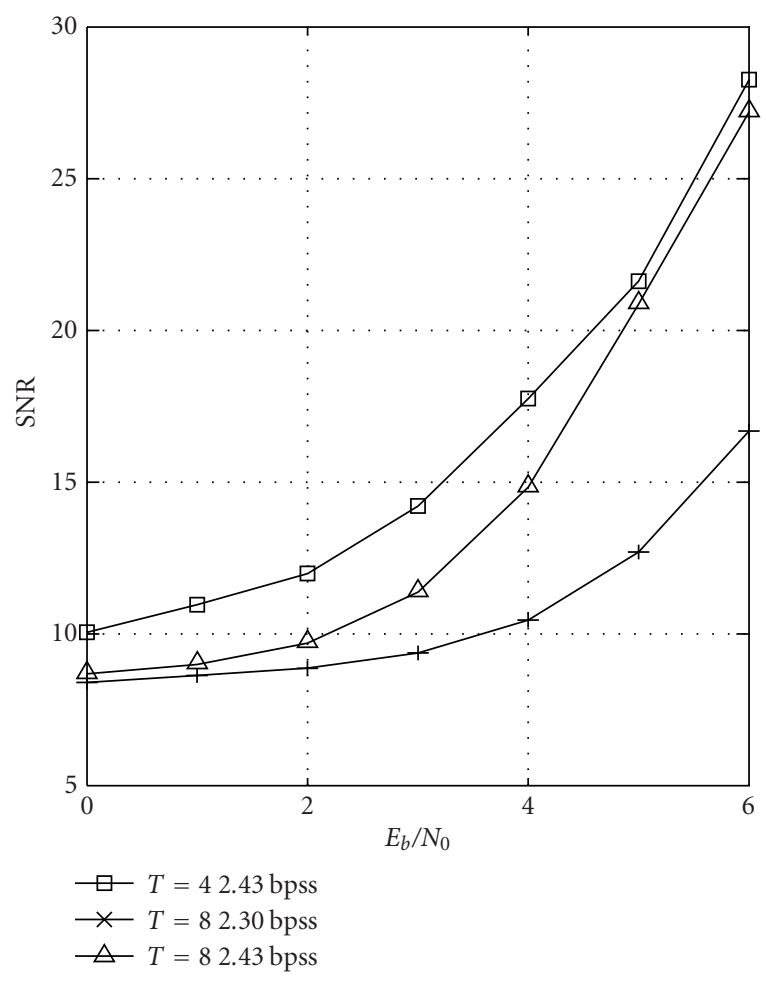

(b)

FIGURE 21: SER and SNR performances of (i) soft quasi-arithmetic decoding $(T=4)$, (ii) soft quasi-arithmetic decoding $(T=8)$, and (iii) soft quasi-arithmetic decoding $(T=8)$ with soft synchronization ( $\rho=0.5,100$ symbols, 3000 channel realizations).

\section{CONCLUSION}

Arithmetic codes are becoming more and more popular in practical compression systems and emerging standards. Their well-known drawback is however their very high sensitivity to noise. MAP estimators running on the coding tree can help to fight against errors and possible decoder desynchronization but at the expense of rather high complexity. The coding tree grows exponentially with the number of symbols in the sequence to be coded. Here, we have considered an alternate solution based on reduced-precision arithmetic codes, called quasi-arithmetic codes. A quasiarithmetic coder can be viewed as a finite-state stochastic automaton. One can then run MAP estimators on the resulting model. For the sake of clarity, we have considered simple source models in the examples. The results reported have been obtained considering an order-1 Markov source. However, the approach extends very easily to higher-order source models. The state model of the coding and decoding process is of finite size. Its size depends on the acceptable approximation of the source distribution. The decoding complexity remains within a realistic range without the need for applying any pruning. Placed in an iterative decoding structure in the spirit of serially concatenated turbo codes, the estimation process can then benefit from the iterations. Overall, the flexibility they offer for adjusting compression efficiency, complexity, and error resilience allows an optimal adaptation to various transmission conditions and terminal capabilities. Notice that, for low complexity, a very good trade-off compression-noise resilience can be achieved with quasi-arithmetic codes for low correlation sources. This emphasizes the interest of the above solution for practical systems, where the coder is applied on quantized decorrelated sequences of symbols.

\section{REFERENCES}

[1] K. P. Subbalakshmi and J. Vaisey, "On the joint sourcechannel decoding of variable-length encoded sources: the BSC case," IEEE Trans. Communications, vol. 49, no. 12, pp. 20522055, 2001.

[2] M. Park and D. J. Miller, "Joint source-channel decoding for variable-length encoded data by exact and approximate MAP sequence estimation," IEEE Trans. Communications, vol. 48, no. 1, pp. 1-6, 2000.

[3] M. Park and D. J. Miller, "Decoding entropy-coded symbols over noisy channels by MAP sequence estimation for asynchronous HMMs," in Proc. Annual Conference on Information Sciences and Systems (CISS '98), pp. 477-482, Princeton, NJ, USA, March 1998.

[4] A. H. Murad and T. E. Fuja, "Joint source-channel decoding of variable length encoded sources," in Proc. Information Theory Workshop (ITW'98), pp. 94-95, Killarney, Ireland, June 1998.

[5] N. Demir and K. Sayood, "Joint source/channel coding for variable length codes," in Proc. IEEE Data Compression Conference (DCC '98), pp. 139-148, Snowbird, Utah, USA, March-April 1998.

[6] R. Bauer and J. Hagenauer, "Turbo FEC/VLC decoding and its application to text compression," in Proc. 34th Annual Conference on Information Sciences and Systems (CISS '00), pp. WA6WA11, Princeton, NJ, USA, March 2000.

[7] A. Guyader, E. Fabre, C. Guillemot, and M. Robert, "Joint 
source-channel turbo decoding of entropy-coded sources," IEEE Journal on Selected Areas in Communications, vol. 19, no. 9, pp. 1680-1696, 2001.

[8] R. Bauer and J. Hagenauer, "Iterative source/channel decoding based on a trellis representation for variable length codes," in Proc. IEEE International Symposium on Information Theory (ISIT '00), p. 117, Sorrento, Italy, June 2000.

[9] Y. Takishima, M. Wada, and H. Murakami, "Reversible variable length codes," IEEE Trans. Communications, vol. 43, no. 4, pp. 158-162, 1995.

[10] J. Wen and J. D. Villasenor, "Reversible variable length codes for efficient and robust image and video coding," in Proc. IEEE Data Compression Conference (DCC '98), pp. 471-480, Snowbird, Utah, USA, March-April 1998.

[11] G. F. Elmasry, "Embedding channel coding in arithmetic coding," IEE Proceedings-Communications, vol. 146, no. 2, pp. 73 $78,1999$.

[12] C. Boyd, J. G. Cleary, S. A. Irvine, I. Rinsma-Melchert, and I. H. Witten, "Integrating error detection into arithmetic coding," IEEE Trans. Communications, vol. 45, no. 1, pp. 1-3, 1997.

[13] G. F. Elmasry, "Joint lossless-source and channel coding using automatic repeat request," IEEE Trans. Communications, vol. 47, no. 7, pp. 953-955, 1999.

[14] I. Sodagar, B. B. Chai, and J. Wus, "A new error resilience technique for image compression using arithmetic coding," in Proc. IEEE Int. Conf. Acoustics, Speech, Signal Processing (ICASSP '00), pp. 2127-2130, Istanbul, Turkey, June 2000.

[15] J. Chou and K. Ramchandran, "Arithmetic coding-based continuous error detection for efficient ARQ-based image transmission," IEEE Journal on Selected Areas in Communications, vol. 18, no. 6, pp. 861-867, 2000.

[16] I. Kozintsev, J. Chou, and K. Ramchandran, "Image transmission using arithmetic coding based continuous error detection," in Proc. IEEE Data Compression Conference (DCC '98), pp. 339-348, Snowbird, Utah, USA, March-April 1998.

[17] B. D. Pettijohn, M. W. Hoffman, and K. Sayood, "Joint source/channel coding using arithmetic codes," IEEE Trans. Communications, vol. 49, no. 5, pp. 826-836, 2001.

[18] C. Demiroglu, M. W. Hoffman, and K. Sayood, "Joint source channel coding using arithmetic codes and trellis coded modulation," in Proc. 11th IEEE Data Compression Conference (DCC '01), pp. 302-311, Snowbird, Utah, USA, March 2001.

[19] T. Guionnet and C. Guillemot, "Soft decoding and synchronization of arithmetic codes: application to image transmission over noisy channels," IEEE Trans. Image Processing, vol. 12, no. 12, pp. 1599-1609, 2003.

[20] P. G. Howard and J. S. Vitter, "Practical implementations of arithmetic coding," in Image and Text Compression, pp. 85112, Kluwer Academic, Norwell, Mass, USA, 1992.

[21] L. R. Bahl, J. Cocke, F. Jelinek, and J. Raviv, "Optimal decoding of linear codes for minimizing symbol error rate," IEEE Transactions on Information Theory, vol. 20, pp. 284-287, March 1974.

[22] J. J. Rissanen, "Generalized Kraft inequality and arithmetic coding," IBM Journal of Research and Development, vol. 20, no. 3, pp. 198-203, 1976.

[23] R. Pasco, Source coding algorithms for fast data compression, Ph.D. thesis, Department of Electrical Engineering, Stanford University, Stanford, Calif, USA, 1976.

[24] J. J. Rissanen, "Arithmetic codings as number representations," Acta Polytechnica Scandinavica, vol. 31, pp. 44-51, December 1979.

[25] I. H. Witten, R. M. Neal, and J. G. Cleary, "Arithmetic coding for data compression," Communications of the ACM, vol. 30, no. 6 , pp. 520-540, 1987.
[26] P. G. Howard and J. S. Vitter, "Design and analysis of fast text compression based on quasi-arithmetic coding," in Proc. IEEE Data Compression Conference (DCC '93), pp. 98-107, Snowbird, Utah, USA, March-April 1993.

Thomas Guionnet received his B.S. degree from the University of Newcastle Upon Tyne, UK, in computer science in 1997. He obtained the Engineer Degree in computer science and image processing and the Ph.D. degree from the University of Rennes 1, France, respectively, in 1999 and 2003. He is currently a Research Engineer at INRIA and is involved in the French national project RNRT VIP and in the JPEG 2000 Part 11JPWL ad hoc group. His research interests include image processing, coding, and joint source and channel coding.

Christine Guillemot is currently "Directeur de Recherche" at INRIA, in charge of a research group dealing with image modelling, processing, and video communication. She holds a Ph.D. degree from École Nationale Supérieure des Télécommunications (ENST) Paris. Her research interests are signal and image processing, coding, and joint source and channel coding. From 1985 to October 1997, she has been with France

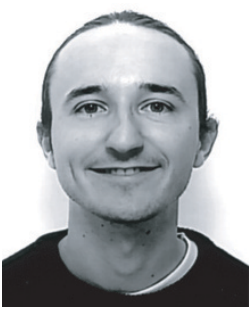
Telecom/CNET at CCETT, where she has been involved in various projects in the domain of coding for TV, HDTV, and multimedia applications. From January 1990 to mid 1991, she has worked at Bellcore, NJ, USA. She serves as an Associate Editor for IEEE Transaction on Image Processing and is a member of the IEEE Image and Multidimensional Signal Processing (IMDSP) committee. 